\title{
Eco-Epidemiological Modelling and Analysis of Prey-Predator Population
}

\author{
Abayneh Fentie Bezabih*, Geremew Kenassa Edessa, Koya Purnachandra Rao \\ Department of Mathematics, Wollega University, Nekemte, Ethiopia \\ Email address: \\ abaynehf2006@gmail.com (A. F. Bezabih), gbonsa.kena@gmail.com (G. K. Edessa), drkpraophd@gmail.com (K. P. Rao) \\ ${ }^{*}$ Corresponding author
}

To cite this article:

Abayneh Fentie Bezabih, Geremew Kenassa Edessa, Koya Purnachandra Rao. Eco-Epidemiological Modelling and Analysis of Prey-

Predator Population. Science Journal of Applied Mathematics and Statistics. Vol. 9, No. 1, 2021, pp. 1-14.

doi: $10.11648 /$ j.sjams.20210901.11

Received: November 10, 2020; Accepted: December 4, 2020; Published: February 23, 2021

\begin{abstract}
In this paper, prey-predator model of five Compartments are constructed with treatment is given to infected prey and infected predator. We took predation incidence rates as functional response type II and disease transmission incidence rates follow simple kinetic mass action function. The positivity, boundedness, and existence of the solution of the model are established and checked. Equilibrium points of the models are identified and Local stability analysis of Trivial Equilibrium point, Axial Equilibrium point, and Disease-free Equilibrium points are performed with the Method of Variation Matrix and Routh Hourwith Criterion. It is found that the Trivial equilibrium point $E_{o}$ is always unstable, and Axial equilibrium point $E_{A}$ is locally asymptotically stable if $\beta k-\left(t_{1}+d_{2}\right)<0, q p_{1} k-d_{3}(s+k)<0, \& q p_{3} k-\left(t_{2}+d_{4}\right)(s+k)<0$ conditions hold true. Global Stability analysis of endemic equilibrium point of the model has been proved by Considering appropriate Liapunove function. In this study, the basic reproduction number of infected prey is obtained to be the following general formula $R_{01}=\left[\left(q p_{1}-d_{3}\right)^{2}\right.$ $\left.k \beta d_{3} s^{2} \zeta\left(q p_{1}-d_{3}\right)\left\{\left(q p_{1}-d_{3}\right)^{2} k s\left(t_{1}+d_{2}\right)+r s q p_{2}\left(k q p_{1}-k d_{3}-d_{3} s\right)\right\}\right]$ and the basic reproduction number of infected predator population is computed and results are written as the general formula of the form as $R_{02}=\left[\left(q p_{1}-d_{3}\right)\left(q p_{3} d_{3}\right) k+\alpha r s q\left(k q p_{1}-k d_{3}-d_{3} s\right)\right]\left(q p_{1}-d_{3}\right)^{2}$ $\left.\left(t_{2}+d_{4}\right) k\right]$. If the basic reproduction number is greater than one, then the disease will persist in prey-predator system. If the basic reproduction number is one, then the disease is stable, and if basic reproduction number less than one, then the disease is dies out from the prey-predator system. Finally, simulations are done with the help of DEDiscover software to clarify results.
\end{abstract}

Keywords: Eco-Epidemiology, Prey-Predator, Stability, Variation Matrix, Reproduction Number, Simulation

\section{Introduction}

Mathematical Modeling of prey-predator systems of interaction of species have a long history since original remarkable work was done by Lotka-Volterra Model in 1920 $[1,3,5,6]$, and SIR model Compartment of systems of population is another vital area of research after pioneering work of Kermack and Mckendrick [1-3, 5-10]. Anderson and May where the first who combined these two modeling systems, while Chattopadhyay and Arino were the first who used the term "eco-epidemiology" for such models [3, 5, 7]. The dynamics of disease in prey-predator systems now become an interesting area of research due to the fact that prey-predator interaction is rich and complex in nature $[4,6$, 7, 11-13]. Several mathematical models have been proposed and studied on prey-predator systems [1-7, 9-12]. Many studies focused on the study of disease in a prey only $[1-5,7$, $12]$, other researchers were interested in the study of disease within the predator population only [14], and there are also some studies on diseases in both prey and predators $[6,9,11]$ In this paper, we proposed and studied infectious disease on both prey and predator interaction of species with treatment given to infected prey and infected predator.

\section{Model Formulation and Assumptions}

In this paper, the prey-predator population divided into five compartments. let us denote $\mathrm{X}(\mathrm{t})$-Susceptible prey, $W(t)$ infected prey, $Y(t)$-Susceptible predator, $Z(t)$ - infected predator, $H(t)$ - both infected prey and infected predator population under treatment. In the absence of infectious disease, the susceptible prey population grows logistically with intrinsic 
growth rate $r$ and environmental carrying capacity $k$ and only susceptible prey can reproduce. In the presence of infectious disease, susceptible predator become infected predator when they come into contact with infected predator, susceptible prey become infected prey when they come into contact with infected prey and the contact process assumed to follow bilinear functional with convolution rate $\alpha, \beta$ respectively. The predation functional response of predator towards the prey assumed to follow a different holling type II functional response form with $p_{1}, \quad p_{2}$ respective predation coefficients of $X(t), W(t)$ due to susceptible predator, and $p_{3}, p_{4}$ respective predation coefficient of $X(t), Y(t)$ due to infected predator. suppose Consumed prey converted into predator with efficiency $q$ and also half saturated constant $s$. It is also assumed that Infected prey $W(t)$ and infected predator $Z(t)$ can only recover through treatment, and treated at treatment rate of $t_{1}, t_{2}$ respectively. The prey-predator population $H(t), W(t), Y(t)$, and $Z(t)$ suffer from infectious disease with death rate $d_{1}, d_{2}, d_{3}$, and $d_{4}$ respectively. Moreover, Assume that all variables and parameters used in the model are non negative.

Table 1. Notations and description of variables.

\begin{tabular}{ll}
\hline Variables & Descriptions \\
\hline $\mathrm{X}(\mathrm{t})$ & Population size of susceptible prey \\
$\mathrm{W}(\mathrm{t})$ & Population size of infectedprey \\
$\mathrm{Y}(\mathrm{t})$ & Population size of susceptible predator \\
$\mathrm{Z}(\mathrm{t})$ & Population size of infectedpredator \\
$H(t)$ & Population size of infected population under treatment \\
\hline
\end{tabular}

Table 2. Notations and Description of parameters.

\begin{tabular}{ll}
\hline parameters & Description of parameters \\
\hline$r, k$ & $\begin{array}{l}\text { Intrinsic growth rate and Carrying capacity of } \\
\text { susceptible prey } \\
\text { disease transmission rates of prey and predator respectively } \\
\text { Treatment rate of infected prey and infected predator } \\
\mathrm{t}_{1}, \mathrm{t}_{2}\end{array}$ \\
$\begin{array}{l}\text { respectively } \\
r_{1}, r_{2}\end{array}$ & $\begin{array}{l}\text { Recovery rate of infected prey and infected predator } \\
\text { respectively }\end{array}$ \\
$p_{i}, i=1,2,3,4$ & $\begin{array}{l}\text { predation coefficients } \\
\text { functional response } \\
f_{i}, i=1,2,3,4\end{array}$ \\
$d_{i}, i=1,2,3,4$ & $\begin{array}{l}\text { death rates } \\
\text { efficiency of predation, and half-saturation constant }\end{array}$ \\
$q, s$ & respectively \\
\hline
\end{tabular}

According to the above assumptions, we have the following Model flow diagram

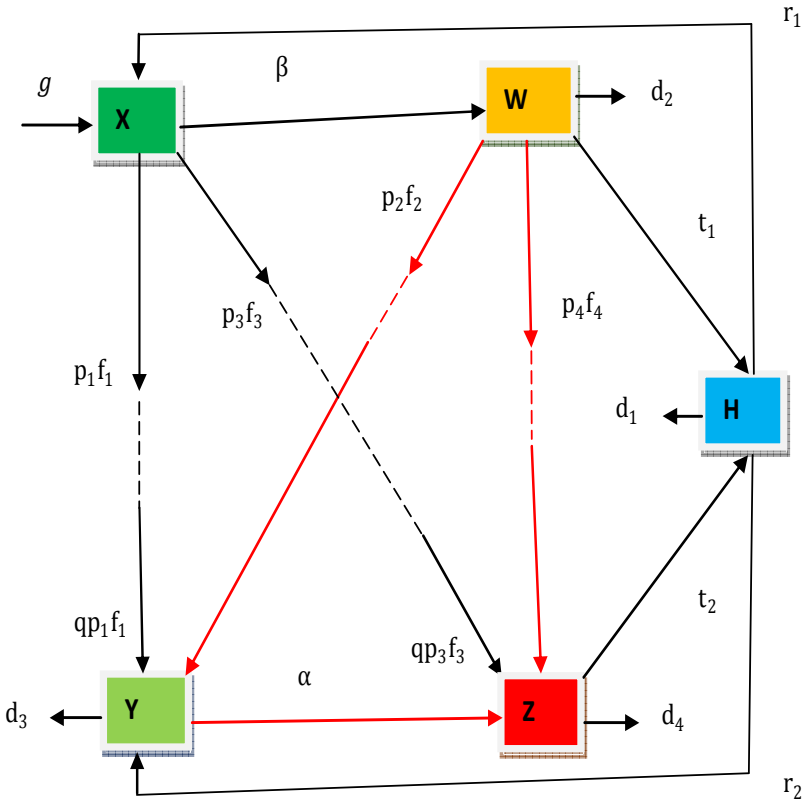

Figure 1. Flow Diagram.

From the Model flow diagram in Figure 1 we have the following set of differential equations

$$
\begin{gathered}
d X / d t=g+r_{1} H-\beta X W-p_{1} f_{1}-p_{3} f_{3} \\
d W / d t=\beta X W-t_{1} W-d_{2} W-p_{2} f_{2}-p_{4} f_{4} \\
d Y / d t=q_{1} f_{1}+q_{2} f_{2}+r_{2} H-\alpha Y Z-d_{3} Y \\
d Z / d t=q_{3} f_{3}+q_{4} f_{4}+\alpha Y Z-t_{2} Z-d_{4} Z \\
d H / d t=t_{1} W+t_{2} Z-d_{1} H-r_{1} H-r_{2} H
\end{gathered}
$$

with initial conditions $\mathrm{X}(0) \geq 0, \mathrm{~W}(0) \geq 0, \mathrm{Y}(0) \geq$ $0, \mathrm{Z}(0) \geq 0, \mathrm{H}(0) \geq 0, \mathrm{p}_{\mathrm{i}}>0, i=1,2,3,4, \& 0<\mathrm{q} \leq 1$

Depending on the assumptions of per capita growth of function $g(X, W)$ for susceptible prey, and different type II functional responses $f_{i}, i=1,2,3,4$.We have more feasible model (6)-(10) emanated from model(1)-(5) as:

$$
\begin{gathered}
\mathrm{dX} / \mathrm{dt}=\mathrm{rX}(1-[\mathrm{X}+\mathrm{W}] / \mathrm{k})+\mathrm{r}_{1} \mathrm{H}-\beta \mathrm{XW}-\left[\mathrm{p}_{1} \mathrm{XY}\right] /[\mathrm{s}+\mathrm{X}]-\left[\mathrm{p}_{3} \mathrm{XZ}\right] /[\mathrm{s}+\mathrm{X}]=\mathrm{f}(\mathrm{X}, \mathrm{W}, \mathrm{Y}, \mathrm{Z}, \mathrm{H}) \\
\mathrm{dW} / \mathrm{dt}=\beta \mathrm{XW}-\mathrm{t}_{1} \mathrm{~W}-\mathrm{d}_{2} \mathrm{~W}-\left[\mathrm{p}_{2} \mathrm{WY}\right] /[\mathrm{s}+\mathrm{W}]-\left[\mathrm{p}_{4} \mathrm{WZ}\right] /[\mathrm{s}+\mathrm{W}]=\mathrm{g}(\mathrm{X}, \mathrm{W}, \mathrm{Y}, \mathrm{Z}, \mathrm{H}) \\
\mathrm{dY} / \mathrm{dt}=\left[\mathrm{qp}_{1} \mathrm{XY}\right] /[\mathrm{s}+\mathrm{X}]+\left[\mathrm{qp}_{2} \mathrm{WY}\right] /[\mathrm{s}+\mathrm{W}]+\mathrm{r}_{2} \mathrm{H}-\alpha \mathrm{YZ}-\mathrm{d}_{3} \mathrm{Y}=\mathrm{h}(\mathrm{X}, \mathrm{W}, \mathrm{Y}, \mathrm{Z}, \mathrm{H}) \\
\mathrm{dZ} / \mathrm{dt}=\left[\mathrm{qp}_{3} \mathrm{XZ}\right] /[\mathrm{s}+\mathrm{X}]+\left[\mathrm{qp}_{4} \mathrm{WZ}\right] /[\mathrm{s}+\mathrm{W}]+\alpha \mathrm{YZ}-\mathrm{t}_{2} \mathrm{Z}-\mathrm{d}_{4} \mathrm{Z}=\mathrm{i}(\mathrm{X}, \mathrm{W}, \mathrm{Y}, \mathrm{Z}, \mathrm{H}) \\
\mathrm{dH} / \mathrm{dt}=\mathrm{t}_{1} \mathrm{~W}+\mathrm{t}_{2} \mathrm{Z}-\mathrm{d}_{1} \mathrm{H}-\mathrm{r}_{1} \mathrm{H}-\mathrm{r}_{2} \mathrm{H}=\mathrm{j}(\mathrm{X}, \mathrm{W}, \mathrm{Y}, \mathrm{Z}, \mathrm{H})
\end{gathered}
$$

with initial conditionsX $(0) \geq 0, W(0) \geq 0, Y(0) \geq 0, Z(0) \geq$ $0, \mathrm{H}(0) \geq 0, \mathrm{p}_{1}, \mathrm{p}_{2}, \mathrm{p}_{3}, \mathrm{p}_{4}>0 \& 0<q \leq 1$

\section{Mathematical Analysis of the Model}

In this section, positivity, boundedness, and existence of the solution of the model is checked. This mathematical analysis of the model could be considered as primarly results.

Theorem 3.1 [Boundedness] All solutions of Model Equations (6)-(10) are bounded in feasible region $\mathbb{R}_{+}^{5}$

Proof: each solutions $X(t), W(t), Y(t), Z(t), H(t)$ of the model is bounded if and only if total population $\mathrm{N}$ is bounded.

Let total population of prey-predator $\mathrm{N}=\mathrm{X}+\mathrm{W}+\mathrm{Y}+$ $\mathrm{Z}+\mathrm{H}$. For $\Lambda>0$ be constant, 


$$
[\mathrm{dN} / \mathrm{dt}]+\Lambda \mathrm{N}=[\mathrm{dX} / \mathrm{dt}]+[\mathrm{dW} / \mathrm{dt}]+[\mathrm{dY} / \mathrm{dt}]+[\mathrm{dZ} / \mathrm{dt}]+[\mathrm{dH} / \mathrm{dt}]+\Lambda \mathrm{N}
$$

By substitute all model Equations (6)-(10)into (11) and removing all negative terms, we have the following results

$[\mathrm{dN} / \mathrm{dt}]+\Lambda \mathrm{N} \leq$ $\mathrm{rX}+\left[\left(\mathrm{qp}_{1} \mathrm{XY}\right) /(\mathrm{s}+\mathrm{X})\right]+\left[\left(\mathrm{qp}_{2} \mathrm{WY}\right) /(\mathrm{s}+\mathrm{W})\right]+$ $\left[\left(\mathrm{qp}_{4} \mathrm{WZ}\right) /(\mathrm{s}+\mathrm{W})\right]+\left[\left(\mathrm{qp}_{3} \mathrm{XZ}\right) /(+\mathrm{X})\right]+\Lambda \mathrm{N}=\mu$. Then $\mathrm{dN} / \mathrm{dt}+\Lambda \mathrm{N} \leq \mu$, Solving the differential inequality, yields:

$\mathrm{N}(\mathrm{t}) \leq[\mu / \Lambda]\left(1-\mathrm{e}^{-\Lambda \mathrm{t}}\right)+\mathrm{N}(0) \mathrm{e}^{-\Lambda \mathrm{t}}$ att $\rightarrow \infty, \mathrm{N} \rightarrow[\mu / \Lambda]$. we know that total prey-predator population is non-negative and hence $0 \leq N(t) \leq[\mu / \Lambda]$ So we have invariant feasible region:

$$
\Omega=\left\{(X, \quad W, \quad Y, \quad Z, \quad H) \in \mathbb{R}_{+}^{5}: 0 \leq \mathrm{N}(\mathrm{t}) \leq[\mu / \Lambda]\right\} .
$$

This prove the theorem and the model is Mathematically well posed

Theorem 3.2[Positivity] All solutions of Model (6)-(10) are non negative.

Proof: To prove theorem 3.2, We have to show that variables $\mathrm{X}(\mathrm{t}), \mathrm{W}(\mathrm{t}), \mathrm{Y}(\mathrm{t}), \mathrm{Z}(\mathrm{t}), \mathrm{H}(\mathrm{t})$ of the Models (6)(10) are non-negative $\forall t \geq 0$.

1. Positivity of $X(\mathrm{t})$ :From the Susceptible prey Model (6), $\mathrm{dX} / \mathrm{dt}=\mathrm{rX}(1-[(\mathrm{X}+\mathrm{W}) / \mathrm{k}])+\mathrm{r}_{1} \mathrm{H}-\beta \mathrm{XW}-$ $\left[\left(p_{1} X Y\right) /(s+X)\right]-\left[\left(p_{3} X Z\right) /(s+X)\right]$ Without loss of generality, After removing all the positive terms from the right hand side of the differential equation, we have the following differential inequality;

$\mathrm{dX} / \mathrm{dt} \geq-\left(\left[\left(\mathrm{rX}^{2}+\mathrm{rXW}\right) / \mathrm{k}\right]+\beta \mathrm{XW}\right.$

$$
\left.+\left[\left(\mathrm{P}_{1} \mathrm{XY}+\mathrm{P}_{3} \mathrm{XZ}\right) /(\mathrm{S}+\mathrm{X})\right]\right)
$$

divide both sides by negative yields:

\section{$-[\mathrm{dX} / \mathrm{dt}] \leq$}

$\left[\left(\mathrm{rX}^{2}+\mathrm{rXW}\right) / \mathrm{k}\right]+\beta \mathrm{XW}+\left[\left(\mathrm{P}_{1} \mathrm{XY}+\mathrm{P}_{3} \mathrm{XZ}\right) /(\mathrm{S}+\mathrm{X})\right]$,

But It is also clear that the following inequality holds

$\left[\left(\mathrm{rX}^{2}+\mathrm{rXW}\right) / \mathrm{k}\right]+\beta \mathrm{XW}+$

$\left[\left(\mathrm{P}_{1} \mathrm{XY}+\mathrm{P}_{3} \mathrm{XZ}\right) /(\mathrm{S}+\mathrm{X})\right] \leq r \mathrm{X}^{2}+\mathrm{rXW}+\beta \mathrm{XW}+$

$\mathrm{p}_{1} \mathrm{XY}+\mathrm{p}_{3} \mathrm{XZ}=\mathrm{X}\left(\mathrm{rX}+\mathrm{rW}+\beta \mathrm{W}+\mathrm{p}_{1} \mathrm{Y}+\mathrm{p}_{3} \mathrm{Z}\right)$

Assume that $r W+\beta W+p_{1} Y+p_{3} Z=C$, Then the

differential inequality reduced to $-[\mathrm{dX} / \mathrm{dt}] \leq$

$\mathrm{X}(\mathrm{rX}+\mathrm{C})$. This inequality can be arranged for integration by partial fraction as $\int[1 /\{\mathrm{X}(\mathrm{rX}+\mathrm{C})\}] \mathrm{dX} \geq$ $\int-\mathrm{d} t$, integrating results

$\int([1 / c] /[X]+[(-r / c)] /[r X+C]) d X \geq-\int d t$, Thus $[1 / C][(\ln |X|) / C]-[1 / C] \ln |\mathrm{rX}+C| \geq-t+Q$, where $\mathrm{Q}$ is integration constant. Using rules of logarithm the inequality can be written as:

$\ln |X /(\mathrm{rX}+\mathrm{C})| \geq-C t+C Q$. Finally solving for $\mathrm{X}$ will give as $X(t) \geq\left[\left(A C e^{-C t}\right) /\left(1-r A e^{-C t}\right)\right]$, where $A=e^{C Q}$. Therefore $X(t)>0$ for $1-r A e^{-C t}>0$. That is $X(t)$ is non-negative for $t>[1 / C] \ln (r A)$

2. Positivity of $W(t)$ : From infected prey Model (7) $\mathrm{dW} / \mathrm{dt}=\beta \mathrm{XW}-\mathrm{t}_{1} \mathrm{~W}-\mathrm{d}_{2} \mathrm{~W}-\left[\left(\mathrm{p}_{2} \mathrm{WY}\right) /(\mathrm{s}+\mathrm{W})\right]-$ $\left[\left(\mathrm{p}_{4} \mathrm{WZ}\right) /(\mathrm{s}+\mathrm{W})\right]$, Without loss of original generality, after removing the positive term $(\beta \mathrm{XW})$. we obtain the following differential inequality,

$d W / d t \geq-\left(\mathrm{t}_{1} \mathrm{~W}+\mathrm{d}_{2} \mathrm{~W}+\left[\left(\mathrm{p}_{2} \mathrm{WY}\right) /(\mathrm{s}+\mathrm{W})\right]+\right.$

$\left.\left[\left(\mathrm{p}_{4} \mathrm{WZ}\right) /(\mathrm{s}+\mathrm{W})\right]\right)$ if

$-\frac{d w}{d t} \leq\left(\mathrm{t}_{1} \mathrm{~W}+\mathrm{d}_{2} \mathrm{~W}+\left[\left(\mathrm{p}_{2} \mathrm{WY}\right) /(\mathrm{s}+\mathrm{W})\right]+\right.$ $\left.\left[\left(\mathrm{p}_{4} \mathrm{WZ}\right) /(\mathrm{s}+\mathrm{W})\right]\right)$, But it is clear that the inequality $\mathrm{t}_{1} \mathrm{~W}+\mathrm{d}_{2} \mathrm{~W}+\left[\left(\mathrm{p}_{2} \mathrm{WY}\right) /(\mathrm{s}+\mathrm{W})\right]+$ $\left[\left(\mathrm{p}_{4} \mathrm{WZ}\right) /(\mathrm{s}+\mathrm{W})\right] \leq \mathrm{t}_{1} \mathrm{~W}+\mathrm{d}_{2} \mathrm{~W}+\mathrm{p}_{2} \mathrm{WY}+\mathrm{p}_{4} \mathrm{WZ}=$ $\left(t_{1}+d_{2}+p_{2} Y+p_{4} Z\right)$ Wholds true. Now Assume thatt ${ }_{1}+$ $\mathrm{d}_{2}+\mathrm{p}_{2} \mathrm{Y}+\mathrm{p}_{4} \mathrm{Z}=\mathrm{C}$. Then we have $-[d W / d t] \leq C \mathrm{~W} t$, Now applying integration yield $\ln |W| \geq-C t+Q$, where $Q$ is integration constant, Then solving for the variable $W(t)$ gives the equation $W(t) \geq e^{-C t+Q}$ which is exponential function and positive at all time. Hence $W(t)$ is positive.

3. Positivity of $Y(t)$ :From Susceptible predator Model (8) $\mathrm{dY} / \mathrm{dt}=\left[\left(\mathrm{qp}_{1} \mathrm{XY}\right) /(\mathrm{s}+\mathrm{X})\right]+\left[\left(\mathrm{qp}_{2} \mathrm{WY}\right) /(\mathrm{s}+\mathrm{W})\right]+$ $\mathrm{r}_{2} \mathrm{H}-\alpha \mathrm{YZ}-\mathrm{d}_{3} \mathrm{Y}$, without loss of original generality, after removing all positive terms $\left(\left[\left(\mathrm{qp}_{1} \mathrm{XY}\right) /(\mathrm{s}+\mathrm{X})\right]+\left[\left(\mathrm{qp}_{2} \mathrm{WY}\right) /(\mathrm{s}+\mathrm{W})\right]+\mathrm{r}_{2} \mathrm{H}\right)$ we obtain differential equation; $\mathrm{dY} / \mathrm{dt} \geq-\left(\alpha \mathrm{z}+\mathrm{d}_{3}\right) \mathrm{y}$, Then applying integration by separable of variable method results, $\ln |y| \geq-\left(\alpha z+d_{3}\right) t+Q$, where $Q$ integration constant and solving for variable $Y(t)$, we obtain the solution $|y| \geq$ $\mathrm{e}^{-\left(\alpha z+d_{3}\right) t+Q}$. Therefore $y(t) \geq \mathrm{e}^{-\left(\alpha z+d_{3}\right) t+Q}$ is a positive exponential function.. hence $y(t)$ is positive.

4. Positivity of $Z(t)$ :From the infected predator Model (9) $\mathrm{dZ} / \mathrm{dt}=\left[\left(\mathrm{qp}_{2} \mathrm{WZ}\right) /(\mathrm{s}+\mathrm{W})\right]+\left[\left(\mathrm{qp}_{3} \mathrm{XZ}\right) /(\mathrm{s}+\mathrm{W})\right]+\alpha \mathrm{YZ}$

$$
-t_{2} Z-d_{4} Z
$$

after removing all positive terms;

$\left(\left[\left(\mathrm{qp}_{2} \mathrm{WZ}\right) /(\mathrm{s}+\mathrm{W})\right]+\left[\left(\mathrm{qp}_{3} \mathrm{XZ}\right) /(\mathrm{s}+\mathrm{W})\right]+\alpha \mathrm{YZ}\right)$, we obtain the differential inequality;

$\mathrm{dZ} / \mathrm{dt} \geq-\left(\mathrm{t}_{2}+\mathrm{d}_{4}\right) \mathrm{z}$

Applying integration by separable of variable method yield

$\ln |z| \geq-\left(\mathrm{t}_{2}+\mathrm{d}_{4}\right) \mathrm{t}+\mathrm{Q}$ where $\mathrm{Q}$ integration constant integration by separable of variable method, Then solving for $Z$ will result $z(t) \geq \mathrm{e}^{-\left(\mathrm{t}_{2}+\mathrm{d}_{4}\right) \mathrm{t}+\mathrm{Q}}$ whichis exponential function that is positive at all time. hence $Z(t)$ is positive

5. Positivity of $\mathrm{H}(\mathrm{t})$ :From infected prey and infected predator population under treatment model (10)

$\mathrm{dH} / \mathrm{dt}=\mathrm{t}_{1} \mathrm{~W}+\mathrm{t}_{2} \mathrm{Z}-\mathrm{d}_{1} \mathrm{H}-\mathrm{r}_{1} \mathrm{H}-\mathrm{r}_{2} \mathrm{H}$, Without loss of generality, after removing all positive terms, we have the differential inequality $\mathrm{dH} / \mathrm{dt} \geq-\left(\mathrm{d}_{1}+\mathrm{r}_{1}+\mathrm{r}_{2}\right) \mathrm{H}$ iff $\mathrm{dH} / \mathrm{H} \geq-\left(\mathrm{d}_{1}+\mathrm{r}_{1}+\mathrm{r}_{2}\right) \mathrm{dt}$ which implies that $\ln |H| \geq$ $-\left(\mathrm{d}_{1}+\mathrm{r}_{1}+\mathrm{r}_{2}\right) \mathrm{t}+\mathrm{Q}$, and solving the variable $H$ provides $|H| \geq e^{-\left(\mathrm{d}_{1}+\mathrm{r}_{1}+\mathrm{r}_{2}\right) \mathrm{t}+\mathrm{Q}}$ is exponential function which is positive at all time. Therefore $(t)>0$, and hence $\mathrm{H}(\mathrm{t})$ is positive. Thus, variables $X(t), W(t), Y(t), Z(t)$ and $H(t)$ are all positive quantities and remain in $\mathbb{R}_{+}^{5}$ for all $t$.

Theorem 3.3 [Existence] All Solutions of the model (6) (10) together with the initial conditions $X(0)>0, W(0) \geq$ $0, Y(0) \geq 0, Z(0) \geq 0, H(0) \geq 0$ exist in $\mathbb{R}_{+}^{5}$ i.e., the model variables $X(t), W(t), Y(t), Z(t)$ and $H(t)$ exist for all $t$ and remain in $\mathbb{R}_{+}^{5}$.

Proof: From the system of differential equation (6)-(10) given as have partial derivatives in the following Table 3 According to Derrick and Groosman theorem, let $\Omega$ denote the region $\Omega=\left\{(X, W, \quad Y, \quad Z, H) \in \mathbb{R}_{+}^{5} ; \mathrm{N} \leq(\mu / \Lambda)\right\}$. Then model (2)-(10) have a unique solution if all partial derivatives of the above functions are continuous and bounded in $\Omega$. 
Here, The continuity and the Boundedness can be shown as follows:

Table 3. Partial derivatives.

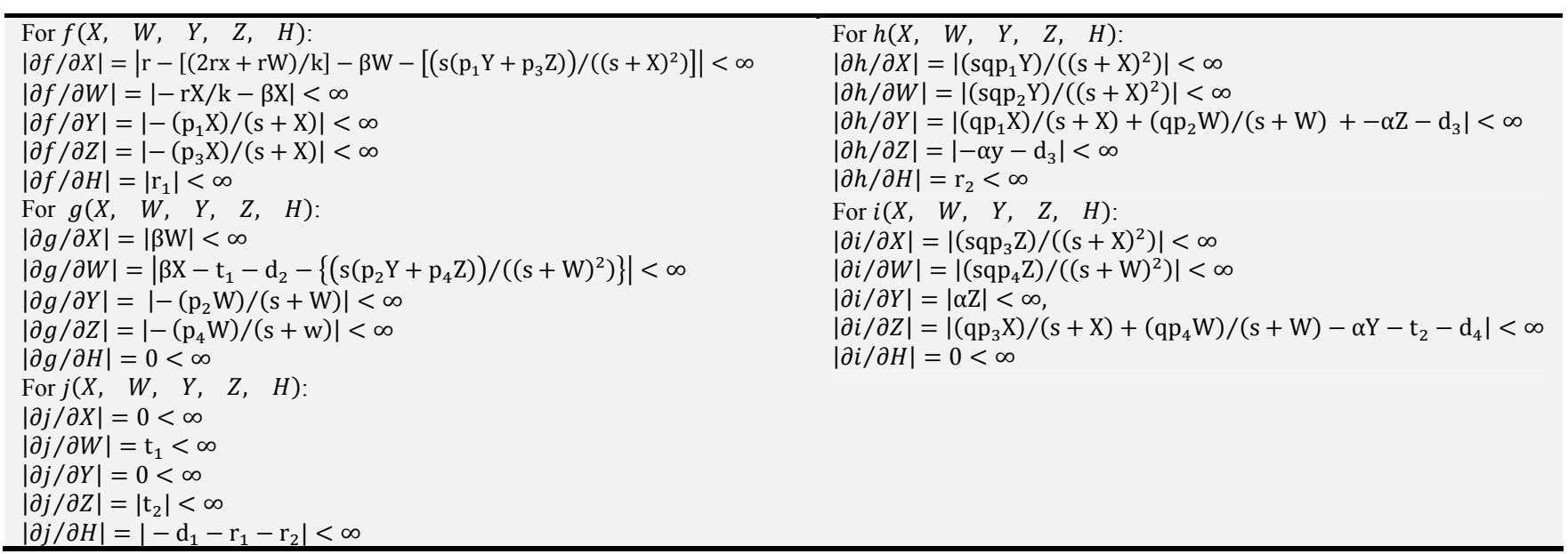

Thus, all the partial derivatives of these functions exist, continuous, and bounded in a region $\Omega$ forallpositive values ofmodel variable and model parameter. Hence, by Derrick and Groosman theorem, a solution for the model (6)-(10) exists and unique.

\section{Stability Analysis}

Stability analysis in the absence of predators in model, That is when $y(t)$ and $Z(t)$ are Zero, model (6)-(10) can be Written as

$$
\begin{gathered}
\mathrm{dX} / \mathrm{dt}=\mathrm{rX}(1-[(\mathrm{X}+\mathrm{W}) / \mathrm{k}])+\mathrm{r}_{1} \mathrm{H}-\beta \mathrm{XW}=\mathrm{f}(\mathrm{X}, \mathrm{W}, \mathrm{H}) \\
\mathrm{dW} / \mathrm{dt}=\beta \mathrm{XW}-\mathrm{t}_{1} \mathrm{~W}-\mathrm{d}_{2} \mathrm{~W}=\mathrm{g}(\mathrm{X}, \mathrm{W}, \mathrm{H}) \\
\mathrm{dH} / \mathrm{dt}=\mathrm{t}_{1} \mathrm{~W}-\mathrm{d}_{1} \mathrm{H}-\mathrm{r}_{1} \mathrm{H}=\mathrm{h}(\mathrm{X}, \mathrm{W}, \mathrm{H})
\end{gathered}
$$

The system (12)-(14) has the following equilibrium points: trivial equilibrium points $E_{o}(0,0,0)$, Axial Equilibrium point $E_{A}(k, 0,0)$ and positive equilibrium point $E_{o}(X, W, H)$ where,

$$
\begin{gathered}
X=k-[(k \beta) / r]-\left[\beta /\left(d_{2}+t_{1}\right)\right] \\
+\left[\left(k \beta r_{1} t_{1}\right) /\left(r\left(d_{1}+r_{1}\right)\left(d_{2}+t_{1}\right)\right)\right], \\
W=\beta /\left(d_{2}+t_{1}\right),
\end{gathered}
$$

$H=\beta /\left[\left(d_{1}+r_{1}\right)\left(d_{2}+t_{1}\right)\right]$, with Next Generation Matrix

$$
=\left(\begin{array}{ccc}
r-[(X, & W, H) \\
\beta W & -[(r X) / k]-\beta X & r_{1} \\
0 & \beta X-t_{1}-d_{2} & 0 \\
& t_{1} & -d_{1}-r_{1}
\end{array}\right)
$$

Theorem 4.1 The Trivial Equilibrium $E_{o}$ isa Saddle point With Unstable manifold in X-direction and Stable Manifold in the WY-plane.
Proof: The Jacobian matrix at $E_{o}$ is given by

$$
J\left(E_{o}\right)=\left(\begin{array}{ccc}
r & 0 & r_{1} \\
0 & -t_{1}-d_{2} & 0 \\
0 & t_{1} & -d_{1}-r_{1}
\end{array}\right)
$$

to compute eigen values compute the $\operatorname{det}\left(J\left(E_{o}\right)-\lambda I_{3}\right)=0$

$$
\left|\begin{array}{ccc}
r-\lambda & 0 & r_{1} \\
0 & -t_{1}-d_{2}-\lambda & 0 \\
0 & t_{1} & -d_{1}-r_{1}-\lambda
\end{array}\right|=0
$$

Then $(r-\lambda)\left(-t_{1}-d_{2}-\lambda\right)\left(-d_{1}-r_{1}-\lambda\right)=0$ is the characteristic polynomial. Thus Eigen Values are: $\lambda_{1}=r>$ $0, \lambda_{2}=-t_{1}-d_{2}<0, \lambda_{3}=-d_{1}-r_{1}<0$ is a saddle point with unstable manifold in X-direction and stable manifold in WY-plane.

Theorem 4.2 The Axial Equilibrium $E_{A}$ is a saddle point if $\beta k-t_{1}-d_{2}>0$ and unstable manifold in $\mathrm{X}$ - direction if $\beta k-t_{1}-d_{2}<0$, then $E_{A}$ stable

Proof: The Jacobian matrix at $E_{A}$ is given by

$$
J\left(E_{A}\right)=\left(\begin{array}{ccc}
-r & -r-\beta k & r_{1} \\
0 & \beta k-t_{1}-d_{2} & 0 \\
0 & t_{1} & -d_{1}-r_{1}
\end{array}\right)
$$

to compute eigen values compute the $\operatorname{det}\left(J\left(E_{o}\right)-\lambda I_{3}\right)=0$

$$
\left|\begin{array}{ccc}
-r-\lambda & -r-\beta k & r_{1} \\
0 & \beta k-t_{1}-d_{2}-\lambda & 0 \\
0 & t_{1} & -d_{1}-r_{1}-\lambda
\end{array}\right|=0
$$

Then $(-r-\lambda)\left(\beta k-t_{1}-d_{2}-\lambda\right)\left(-d_{1}-r_{1}-\lambda\right)=0$ is characteristic polynomial. Thus $\lambda_{1}=-r<0, \lambda_{2}=\beta k-t_{1}-$ $d_{2}, \lambda_{3}=-d_{1}-r_{1}<0$, hence Axial equilibrium point is saddle point if $\beta k-t_{1}-d_{2}>0$ and stable if $\beta k-t_{1}-d_{2}<0$

Stability Analysis in the absence of infectious Disease in the $\operatorname{system}(2)$ That is When there is no disease $(\mathrm{t}), \mathrm{Z}(\mathrm{t})$ and $\mathrm{H}(\mathrm{t})$ are all zero and Model(6)-(10) becomes 


$$
\begin{array}{r}
d X / d t=r X(1-[(X+W) / k])-\left[\left(p_{1} X Y\right) /(s+X)\right]=f(X, Y) \\
d Y / d t=\left[\left(q_{1} p_{1} X\right) /(s+X)\right]-d_{3} Y=g(X, Y)
\end{array}
$$

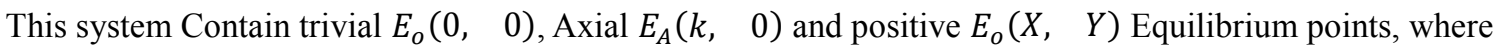

$$
\begin{gathered}
X=\left\{\left[r(k-s)+\sqrt{r} \sqrt{k^{2} r+2 k r s+r X s^{2}-4 k p_{1}}\right] /[2 r]\right\} \\
Y=\left\{\left(q p_{1}\right) /\left(d_{3}\left(s+\left[\left(r(k-s)+\sqrt{r} \sqrt{k^{2} r+2 k r s+r s^{2}-4 k p_{1}}\right) /(2 r)\right]\right)\right)\right\}
\end{gathered}
$$

with Jacobian matrix is given by

$$
J(X, Y)=\left(\begin{array}{cc}
r-[(2 r X) / k]-\left[\left(p_{1} Y S\right) /\left((s+X)^{2}\right)\right] & {\left[q p_{1} X\right] /[s+k]} \\
{\left[q p_{1} Y S\right] /\left[(s+X)^{2}\right]} & {\left[q p_{1} X\right] /[s+X]-d_{3}}
\end{array}\right)
$$

Theorem 4.4The trivial equilibrium $E_{o}$ isa saddle point with unstable manifold in X-direction and stable manifold in Ydirection

Proof: The Jacobian matrix at $E_{o}$ is given by

$$
J\left(E_{o}\right)=\left(\begin{array}{cc}
r & 0 \\
0 & -d_{3}
\end{array}\right)
$$

hence eigen values are: $\lambda_{1}=r>0, \lambda_{2}=-d_{3}<0$ which is a saddle point.

Theorem 4.5 The axial equilibrium $E_{A}$ is stable if $\frac{q p_{1} k}{s+k}-d_{3}<0$,otherwise unstable proof: The Jacobian Matrix at $E_{A}$ is given by

$$
J\left(E_{A}\right)=\left(\begin{array}{cc}
-r & -\left[p_{1} k\right] /[s+k] \\
0 & -\left[p_{1} k\right] /[s+k]-d_{3}
\end{array}\right)
$$

To find eigen values compute $\operatorname{det}\left(J\left(E_{A}\right)-\lambda I_{3}\right)=0$

$$
\left|\begin{array}{cc}
-r-\lambda & -\left[p_{1} k\right] /[s+k] \\
0 & {\left[q p_{1} k\right] /[s+k]-d_{3}-\lambda}
\end{array}\right|=0
$$

Eigen values are: $\lambda_{1}=-r<0, \lambda_{2}=\left[q p_{1} k\right] /[s+k]-d_{3}$

Thus $E_{A}$ is stable if $\frac{q p_{1} k}{s+k}-d_{3}<0$ andotherwise unstable.

Theorem 4.6 The positive equilibrium $E$ isstable if $\left\{r-(2 r X) / k-\left[p_{1} Y S\right] /\left[(s+X)^{2}\right]\right\}+\left\{\left[q p_{1} k\right] /[s+k]-d_{3}\right\}>0$ and $\left\{r-(2 r X) / k-\left[p_{1} Y S\right] /\left[(s+X)^{2}\right]\right\} *\left\{\left[q p_{1} k\right] /[s+k]-d_{3}\right\}+\left\{\left[q p_{1}{ }^{2} X Y S\right] /\left[(s+X)^{3}\right]\right\}>0$

proof: The Jacobian Matrix at $E$ is given by

$$
J(X, Y)=\left(\begin{array}{cc}
r-[2 r X] / k-\left[p_{1} Y S\right] /(s+X)^{2} & {\left[q p_{1} X\right] /[s+k]} \\
{\left[q p_{1} Y S\right] /\left[(s+X)^{2}\right]} & {\left[q p_{1} X\right] /[s+X]-d_{3}}
\end{array}\right)
$$

Then compute $\operatorname{det}\left(J(E)-\lambda I_{3}\right)=0$

$$
\left|\begin{array}{cc}
r-[2 r X] / k-\left[p_{1} Y S\right] /\left[(s+X)^{2}\right]-\lambda & {\left[q p_{1} X\right] /[s+k]} \\
{\left[q p_{1} Y S\right] /\left[(s+X)^{2}\right]} & {\left[q p_{1} X\right] /[s+X]-d_{3}-\lambda}
\end{array}\right|=0
$$

Then

$$
(\underbrace{r-[(2 r X) / k]-\left[\left(p_{1} Y S\right) /\left((s+X)^{2}\right)\right]}_{a}-\lambda)(\underbrace{\left[\left(q p_{1} X\right) /(s+X)\right]-d_{3}}_{b}-\lambda)+\underbrace{\left[\left(s q p_{1}{ }^{2} X Y\right) /\left((s+X)^{3}\right)\right]}_{c}=0
$$

is characteristic polynomial. Using Routh Hourwith criterion the quadratic polynomial is stable if $a+b>0, a b+c>0$ otherwise unstable. Equilibrium points Model(2)-(10) are Steady state points of the form $(X, W, \quad Y, Z, \quad H)$ ofmodel (2) that satisfiesdX/dt $=\mathrm{dW} / \mathrm{dt}=\mathrm{dY} / \mathrm{dt}=\mathrm{dZ} / \mathrm{dt}=\mathrm{dH} / \mathrm{dt}=0$, provided that each variable is non-negative. In Model(2) Five steady state points are identified and listed here: trivial steady state $\mathrm{E}_{\mathrm{o}}(0,0,0,0,0)$, Axial steady state $\mathrm{E}_{\mathrm{A}}(\mathrm{k}, 0,0,0,0)$, Disease-free steady state $\bar{E}(\bar{X}, 0, \quad \bar{Y}, 0,0)$ and endemic steady state $\mathrm{E}^{*}\left(\mathrm{X}^{*}, \mathrm{~W}^{*}, \mathrm{Y}^{*}, \mathrm{Z}^{*}, \mathrm{H}^{*}\right)$. computation of disease free and endemic equilibrium points are presented as follows:

Disease free equilibrium points[DFEP] of model(2)-(10) are steady state Solutions when there is no infectious disease in the population. In the absence of infectious disease in prey-predator system the variables $W(t)=Z(t)=H(t)=0$ anddX/dt $=$ $\mathrm{dW} / \mathrm{dt}=\mathrm{dY} / \mathrm{dt}=\mathrm{dZ} / \mathrm{dt}=\mathrm{dH} / \mathrm{dt}=0$, Then model(2)-(10) become 


$$
\left\{\begin{array}{c}
r \bar{X}(1-[\bar{X} / k])-\left[\left(p_{1} \bar{X} \bar{Y}\right) /(s+X)\right]=0 \\
{\left[\left(q p_{1} \bar{X} \bar{Y}\right) /(s+\bar{X})\right]-d_{3} \bar{Y}=0}
\end{array}\right.
$$

Thus solving $\bar{X}$ and $\bar{Y}$,it is found that

$$
\bar{X}=\left\{\left(\mathrm{d}_{3} \mathrm{~s}\right) /\left(\mathrm{qp}_{1}-\mathrm{d}_{3}\right)\right\} \& \bar{Y}=\left\{\left(\mathrm{rsq}\left(\mathrm{kqp}_{1}-\mathrm{kd}_{3}-\mathrm{d}_{3} \mathrm{~s}\right)\right) /\left(\left(\mathrm{qp}_{1}-\mathrm{d}_{3}\right)^{2} \mathrm{k}\right)\right\} \text {, and }
$$

Hence disease-free equilibrium point(DFEP) of Model (2)is given by

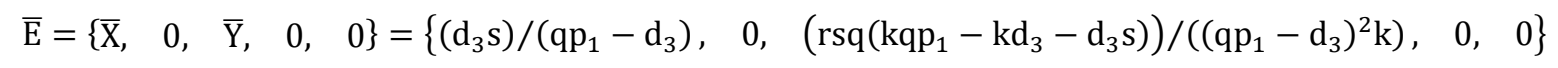

The endemic equilibrium point[EEP] is positive equilibrium point $\mathrm{E}^{*}\left(\mathrm{X}^{*}, \mathrm{~W}^{*}, \mathrm{Y}^{*}, \mathrm{Z}^{*}, \mathrm{H}^{*}\right)$ obtained by solving modelequation (2) as $\mathrm{dX} / \mathrm{dt}=\mathrm{dW} / \mathrm{dt}=\mathrm{dY} / \mathrm{dt}=\mathrm{dZ} / \mathrm{dt}=\mathrm{dH} / \mathrm{dt}=0$ for which all variables are non zero

$$
\left\{\begin{array}{c}
\mathrm{rX}\left(1-\left[\left(\mathrm{X}^{*}+\mathrm{W}^{*}\right) / \mathrm{k}\right]\right)+\mathrm{r}_{1} \mathrm{H}^{*}-\beta \mathrm{X}^{*} \mathrm{~W}^{*}-\left[\left(\mathrm{p}_{1} \mathrm{X}^{*} \mathrm{Y}^{*}+\mathrm{p}_{3} \mathrm{X}^{*} \mathrm{Z}^{*}\right) /\left(\mathrm{s}+\mathrm{X}^{*}\right)\right]=0 \\
\beta \mathrm{X}^{*} \mathrm{~W}^{*}-\mathrm{t}_{1} \mathrm{~W}^{*}-\mathrm{d}_{2} \mathrm{~W}^{*}-\left[\left(\mathrm{p}_{2} \mathrm{~W}^{*} \mathrm{Y}^{*}+\mathrm{p}_{4} \mathrm{~W}^{*} \mathrm{Z}^{*}\right) /\left(\mathrm{s}+\mathrm{W}^{*}\right)\right]=0 \\
{\left[\left(\mathrm{qp}_{1} \mathrm{X}^{*} \mathrm{Y}^{*}\right) /\left(\mathrm{s}+\mathrm{X}^{*}\right)\right]+\left[\left(\mathrm{qp}_{2} \mathrm{~W}^{*} \mathrm{Y}^{*}\right) /\left(\mathrm{s}+\mathrm{W}^{*}\right)\right]+\mathrm{r}_{2} \mathrm{H}^{*}-\alpha \mathrm{Y}^{*} \mathrm{Z}^{*}-\mathrm{d}_{3} \mathrm{Y}^{*}=0} \\
{\left[\left(\mathrm{qp}_{3} \mathrm{X}^{*} \mathrm{Z}^{*}\right) /\left(\mathrm{s}+\mathrm{X}^{*}\right)\right]+\left[\left(\mathrm{qp}_{4} \mathrm{~W}^{*} \mathrm{Z}^{*}\right) /\left(\mathrm{s}+\mathrm{W}^{*}\right)\right]+\alpha \mathrm{Y}^{*} \mathrm{Z}^{*}-\left(\mathrm{t}_{2}+\mathrm{d}_{4}\right) \mathrm{Z}^{*}=0} \\
\mathrm{t}_{1} \mathrm{~W}^{*}+\mathrm{t}_{2} \mathrm{Z}^{*}-\left(\mathrm{d}_{1}+\mathrm{r}_{1}+\mathrm{r}_{2}\right) \mathrm{H}^{*}=0
\end{array}\right.
$$

Then solving for the variables $\mathrm{X}^{*}, \mathrm{~W}^{*}, \mathrm{Y}^{*}, \mathrm{Z}^{*}, \& H^{*}$, the endemic equilibrium points of the model exists, and a simplified result obtained as:

$$
\begin{gathered}
X^{*}=\left\{-r \beta d_{1}+[2 k r-r s-2 k \beta] d_{1} d_{2}-r \beta r_{1}+2 k r d_{2} r_{1}\right\} \\
\mathrm{W}^{*}=\left\{\left[\beta-\mathrm{s}\left(\mathrm{d}_{2}+\mathrm{t}_{1}\right)-\sqrt{4\left(\mathrm{~s} \beta-\mathrm{p}_{2}-\mathrm{p}_{4}\right)\left(\mathrm{t}_{1}+\mathrm{d}_{2}\right)+\left(\mathrm{s}\left[\mathrm{t}_{1}+\mathrm{d}_{2}\right]-\beta\right)^{2}}\right] /\left[2\left(\mathrm{t}_{1}+\mathrm{d}_{2}\right)\right]\right\} \\
Y^{*}=\left\{-\alpha+\left[\left(\beta-s\left(d_{2}+t_{1}\right)\right) r r_{2}\right] /\left[2 k r_{1} d_{3}\left(t_{1}+d_{2}\right)\right]\right\} \\
Z^{*}=\left\{\left(\alpha+\left[q p_{4}\right] /\left[s+\left(\beta-\left(t_{1}+d_{2}\right)+\sqrt{4\left(s \beta-p_{2}-p_{4}\right)\left(t_{1}+d_{2}\right)+\left(s\left[\mathrm{t}_{1}+\mathrm{d}_{2}\right]-\beta\right)^{2}}\right) /\left(2\left(t_{1}+d_{2}\right)\right)\right]\right) /\left(t_{2}+d_{4}\right)\right\} \\
\mathrm{H}^{*}=\left\{\left(\beta-r+\left[r \beta-r s d_{2}-r s t_{1}\right] /\left[2 k\left(t_{1}+d_{2}\right)\right]\right) / r_{1}\right\}
\end{gathered}
$$

To study the Stability analysis of equilibrium points of model (6)-(10), it is better to linearize mode (6)-(10) using Variation matrix. Then the Variation Matrix of these functions (6)-(10)is given by

$$
\mathrm{V}(\mathrm{X}, \mathrm{W}, \mathrm{Y}, \mathrm{Z}, \mathrm{H})=\left(\begin{array}{ccccc}
\mathrm{f}_{\mathrm{X}} & \mathrm{f}_{\mathrm{W}} & \mathrm{f}_{\mathrm{Y}} & \mathrm{f}_{\mathrm{Z}} & \mathrm{f}_{\mathrm{H}} \\
\mathrm{g}_{\mathrm{X}} & \mathrm{g}_{\mathrm{W}} & \mathrm{g}_{\mathrm{Y}} & \mathrm{g}_{\mathrm{Z}} & \mathrm{g}_{\mathrm{H}} \\
\mathrm{h}_{X} & \mathrm{~h}_{\mathrm{W}} & \mathrm{h}_{\mathrm{Y}} & \mathrm{h}_{\mathrm{Z}} & \mathrm{h}_{\mathrm{H}} \\
\mathrm{i}_{\mathrm{X}} & \mathrm{i}_{\mathrm{W}} & \mathrm{i}_{\mathrm{Y}} & \mathrm{i}_{\mathrm{Z}} & \mathrm{i}_{\mathrm{H}} \\
\mathrm{j}_{\mathrm{X}} & \mathrm{j}_{\mathrm{W}} & \mathrm{j}_{\mathrm{Y}} & \mathrm{j}_{\mathrm{Z}} & \mathrm{j}_{\mathrm{H}}
\end{array}\right)
$$

Where each element of the matrix represent partial derivatives of functions (6)-(10) with respect to model variables, and Computations of each element of the variation matrix given as:

$$
\mathrm{V}(\mathrm{X}, \mathrm{W}, \mathrm{Y}, \mathrm{Z}, \mathrm{H})=\left(\begin{array}{ccccc}
\mathrm{f}_{\mathrm{X}} & -(\mathrm{rX}) / \mathrm{k}-\beta \mathrm{X} & -\left(\mathrm{p}_{1} \mathrm{X}\right) /(\mathrm{s}+\mathrm{X}) & -\left(\mathrm{p}_{3} \mathrm{X}\right) /(\mathrm{s}+\mathrm{X}) & \mathrm{r}_{1} \\
\beta \mathrm{W} & \mathrm{g}_{\mathrm{W}} & -\left(\mathrm{p}_{2} \mathrm{~W}\right) /(\mathrm{s}+\mathrm{W}) & -\left(\mathrm{p}_{4} \mathrm{~W}\right) /(\mathrm{s}+\mathrm{w}) & 0 \\
{\left[\mathrm{sqp}_{1} \mathrm{Y}\right] /\left[(\mathrm{s}+\mathrm{X})^{2}\right]} & {\left[\mathrm{sqp}_{2} \mathrm{Y}\right] /\left[(\mathrm{s}+\mathrm{X})^{2}\right]} & \mathrm{h}_{\mathrm{Y}} & -\alpha \mathrm{y}-\mathrm{d}_{3} & \mathrm{r}_{2} \\
{\left[\mathrm{sqp}_{3} \mathrm{Z}\right] /\left[(\mathrm{s}+\mathrm{X})^{2}\right]} & {\left[\mathrm{sqp}_{4} \mathrm{Z}\right] /\left[(\mathrm{s}+\mathrm{W})^{2}\right]} & \alpha \mathrm{Z} & \mathrm{i}_{\mathrm{z}} & 0 \\
0 & \mathrm{t}_{1} & 0 & \mathrm{t}_{2} & -\mathrm{d}_{1}-\mathrm{r}_{1}-\mathrm{r}_{2}
\end{array}\right)
$$

where

$$
\begin{gathered}
\mathrm{f}_{\mathrm{X}}=\mathrm{r}-[(2 \mathrm{rx}) / \mathrm{k}]-[(\mathrm{rW}) / \mathrm{k}]-\beta \mathrm{W}-\left\{\left[\mathrm{s}\left(\mathrm{p}_{1} \mathrm{Y}+\mathrm{p}_{3} \mathrm{Z}\right)\right] /\left[(\mathrm{s}+\mathrm{X})^{2}\right]\right\}, \\
\mathrm{g}_{\mathrm{W}}=\beta \mathrm{X}-\mathrm{t}_{1}-\mathrm{d}_{2}-\left\{\left[\mathrm{s}\left(\mathrm{p}_{2} \mathrm{Y}+\mathrm{p}_{4} \mathrm{Z}\right)\right] /\left[(\mathrm{s}+\mathrm{W})^{2}\right]\right\}, \\
\mathrm{h}_{\mathrm{Y}}=\left\{\left(\mathrm{qp}_{1} \mathrm{X}\right) /(\mathrm{s}+\mathrm{X})\right\}+\left\{\left[\mathrm{qp}_{2} \mathrm{~W}\right] /[\mathrm{s}+\mathrm{W}]\right\}-\alpha \mathrm{Z}-\mathrm{d}_{3}, \\
\mathrm{i}_{\mathrm{z}}=\left\{\left[\mathrm{qp}_{3} \mathrm{X}\right] /[\mathrm{s}+\mathrm{X}]\right\}+\left\{\left[\mathrm{qp}_{4} \mathrm{~W}\right] /[\mathrm{s}+\mathrm{W}]\right\}-\alpha \mathrm{Y}-\mathrm{t}_{2}-\mathrm{d}_{4},
\end{gathered}
$$

Theorem 4.7[TEP] Trivial equilibrium point $\mathrm{E}_{\mathrm{o}}(0,0,0,0,0)$ is always locally asymptotically unstable. 
Proof: Consider the Variation Matrix (17) at $\mathrm{E}_{\mathrm{o}}$

$$
\mathrm{V}\left(\mathrm{E}_{\mathrm{o}}\right)=\left(\begin{array}{ccccc}
\mathrm{r} & 0 & 0 & 0 & \mathrm{r}_{1} \\
0 & -\mathrm{t}_{1}-d_{2} & 0 & 0 & 0 \\
0 & 0 & -d_{3} & 0 & \mathrm{r}_{2} \\
0 & 0 & 0 & -\mathrm{t}_{2}-d_{4} & 0 \\
0 & \mathrm{t}_{1} & 0 & \mathrm{t}_{2} & -d_{1}-\mathrm{r}_{1}-\mathrm{r}_{2}
\end{array}\right)
$$

Eigen value of variation matrix can be computed from the characteristic polynomial $\operatorname{det}\left(\mathrm{V}\left(\mathrm{E}_{\mathrm{o}}\right)-\lambda I_{5}\right)=0$

$$
\left|\begin{array}{ccccc}
\mathrm{r}-\lambda & 0 & 0 & 0 & \mathrm{r}_{1} \\
0 & -\mathrm{t}_{1}-d_{2}-\lambda & 0 & 0 & 0 \\
0 & 0 & -d_{3}-\lambda & 0 & \mathrm{r}_{2} \\
0 & 0 & 0 & -\mathrm{t}_{2}-d_{4}-\lambda & 0 \\
0 & \mathrm{a}_{1} & 0 & \mathrm{a}_{2} & -d_{1}-\mathrm{r}_{1}-\mathrm{r}_{2}-\lambda
\end{array}\right|=0
$$

$\Rightarrow(\mathrm{r}-\lambda)\left(-\mathrm{t}_{1}-d_{2}-\lambda\right)\left(-d_{3}-\lambda\right)\left(-\mathrm{t}_{2}-d_{4}-\lambda\right)\left(-d_{1}-\mathrm{r}_{1}-\mathrm{r}_{2}-\lambda\right)=0$ is the characteristic polynomial.

The eigen values are:

$\lambda_{1}=\mathrm{r}>0, \lambda_{2}=-\mathrm{t}_{1}-d_{2}<0, \lambda_{3}=-d_{3}<0, \lambda_{4}=-\mathrm{t}_{2}-d_{4}<0, \lambda_{5}=-d_{1}-\mathrm{r}_{1}-\mathrm{r}_{2}<0$, Thus the trivial equilibrium point is a saddle point with locally asymptotically unstable manifold in X-direction, and locally asymptotically stable manifold in $W, Y, Z, H$ directions.

Theorem 4.8[AEP] Axial Equilibrium Point $\mathrm{E}_{\mathrm{A}}(\mathrm{k}, 0,0,0,0)$ exists and always locally asymptotically stable in model(2)if and only if model parameters satisfy the conditions: $\beta \mathrm{k}-\mathrm{t}_{1}-d_{2}<0,\left[\left(\mathrm{qp}_{1} \mathrm{k}\right) /(\mathrm{s}+\mathrm{k})\right]-d_{3}<0, \&\left[\left(\mathrm{qp}_{3} \mathrm{k}\right) /(\mathrm{s}+\mathrm{k})\right]-\mathrm{t}_{2}-$ $d_{4}<0$. otherwise $\mathrm{E}_{A}$ is locally asymptotically unstable.

Proof: Consider the Variation matrix (17) at $\mathrm{J}\left(\mathrm{E}_{\mathrm{A}}\right)$

$$
\mathrm{V}\left(\mathrm{E}_{\mathrm{A}}\right)=\left(\begin{array}{ccccc}
-\mathrm{r} & -\mathrm{r}-\beta \mathrm{k} & -\left[\left(\mathrm{p}_{1} \mathrm{k}\right) /(\mathrm{s}+\mathrm{k})\right] & -\left[\left(\mathrm{p}_{3} \mathrm{k}\right) /(\mathrm{s}+\mathrm{k})\right] & \mathrm{r}_{1} \\
0 & \beta \mathrm{k}-\mathrm{t}_{1}-d_{2} & 0 & 0 & 0 \\
0 & 0 & {\left[\left(\mathrm{qp}_{1} \mathrm{k}\right) /(\mathrm{s}+\mathrm{k})\right]-d_{3}} & \mathrm{~d}_{3} & \mathrm{r}_{2} \\
0 & 0 & 0 & {\left[\left(\mathrm{qp}_{3} \mathrm{k}\right) /(\mathrm{s}+\mathrm{k})\right]-\mathrm{t}_{2}-d_{4}} & 0 \\
0 & \mathrm{t}_{1} & 0 & \mathrm{t}_{2} & -d_{1}-\mathrm{r}_{1}-\mathrm{r}_{2}
\end{array}\right)
$$

Then it is possible to find eigen value from characteristic matrix as $\operatorname{Det}\left(\mathrm{V}\left(\mathrm{E}_{\mathrm{A}}\right)-\lambda \mathrm{I}_{5}\right)$

$$
\begin{gathered}
\begin{array}{ccccc}
-\mathrm{r}-\lambda & -\mathrm{r}-\beta \mathrm{k} & -\left[\left(\mathrm{p}_{1} \mathrm{k}\right) /(\mathrm{s}+\mathrm{k})\right] & -\left[\left(\mathrm{p}_{3} \mathrm{k}\right) /(\mathrm{s}+\mathrm{k})\right] & \mathrm{r}_{1} \\
0 & \beta \mathrm{k}-\mathrm{t}_{1}-d_{2}-\lambda & 0 & 0 & 0 \\
0 & 0 & {\left[\left(\mathrm{qp}_{1} \mathrm{k}\right) /(\mathrm{s}+\mathrm{k})\right]-d_{3}-\lambda} & \mathrm{d}_{3} & \mathrm{r}_{2} \\
0 & 0 & 0 & {\left[\left(\mathrm{qp}_{3} \mathrm{k}\right) /(\mathrm{s}+\mathrm{k})\right]-\mathrm{t}_{2}-d_{4}-\lambda} & 0 \\
0 & \mathrm{t}_{1} & 0 & \mathrm{t}_{2} & -d_{1}-\mathrm{r}_{1}-\mathrm{r}_{2}-\lambda
\end{array} \mid \\
(-\mathrm{r}-\lambda)\left(\beta \mathrm{k}-\mathrm{t}_{1}-d_{2}-\lambda\right)\left(\left[\left(\mathrm{qp}_{1} \mathrm{k}\right) /(\mathrm{s}+\mathrm{k})\right]-d_{3}-\lambda\right)\left(\left[\left(\mathrm{qp}_{3} \mathrm{k}\right) /(\mathrm{s}+\mathrm{k})\right]-\mathrm{t}_{2}-d_{4}-\lambda\right)\left(-d_{1}-\mathrm{r}_{1}-\mathrm{r}_{2}-\lambda\right)
\end{gathered}
$$

is characteristic polynomial. Then Eigen values are roots of this polynomial

$\lambda_{1}=-\mathrm{r}<0, \lambda_{2}=-d_{1}-\mathrm{r}_{1}-\mathrm{r}_{2}<0, \lambda_{3}=\beta \mathrm{k}-\mathrm{t}_{1}-d_{2}, \lambda_{4}=\left[\left(\mathrm{qp}_{1} \mathrm{k}\right) /(\mathrm{s}+\mathrm{k})\right]-d_{3}, \lambda_{5}=\left[\left(\mathrm{qp} \mathrm{p}_{3} \mathrm{k}\right) /(\mathrm{s}+\mathrm{k})\right]-\mathrm{t}_{2}-d_{4}$

The Axial equilibrium point $\mathrm{E}_{A}$ is locally asymptotically stable, if

otherwise $\mathrm{E}_{A}$ is locally asymptotically unstable.

$$
\beta \mathrm{k}-\mathrm{t}_{1}-d_{2}<0,\left[\left(\mathrm{qp}_{1} \mathrm{k}\right) /(\mathrm{s}+\mathrm{k})\right]-d_{3}<0, \&\left[\left(\mathrm{qp}_{3} \mathrm{k}\right) /(\mathrm{s}+\mathrm{k})\right]-\mathrm{t}_{2}-d_{4}<0
$$

Theorem 4.9 [DFEP] The disease- free equilibrium point

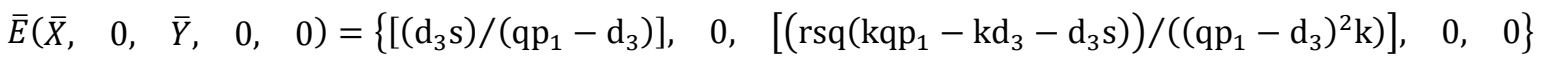

exists and always locally asymptotically stable if and only if the model parameter satisfy conditions:

$$
\begin{gathered}
\beta \overline{\mathrm{X}}-\mathrm{t}_{1}-\mathrm{d}_{2}-\left[\left(\mathrm{sp}_{2} \overline{\mathrm{Y}}\right) /\left(\mathrm{s}^{2}\right)\right] \leq 0,\left[\left(\mathrm{qp}_{3} \overline{\mathrm{X}}\right) /(\mathrm{s}+\mathrm{X})\right]-\alpha \overline{\mathrm{Y}}-\mathrm{t}_{2}-\mathrm{d}_{4} \leq 0 \text { and }\left\{\mathrm{r}-[(2 \mathrm{r} \overline{\mathrm{X}}) / \mathrm{k}]-\left[\left(\mathrm{sp}_{1} \overline{\mathrm{Y}}\right) /\left((\mathrm{s}+\overline{\mathrm{X}})^{2}\right)\right]\right\}+ \\
\left\{\left[\left(\mathrm{qp}_{1} \overline{\mathrm{X}}\right) /(\mathrm{s}+\overline{\mathrm{X}})\right]-\mathrm{d}_{3}\right\}>0 \\
\&\left\{\mathrm{r}-[(2 \mathrm{r} \overline{\mathrm{X}}) / \mathrm{k}]-\left[\left(\mathrm{sp}_{1} \overline{\mathrm{Y}}\right) /\left((\mathrm{s}+\overline{\mathrm{X}})^{2}\right)\right]\right\} *\left\{\left[\left(\mathrm{qp}_{1} \overline{\mathrm{X}}\right) /(\mathrm{s}+\overline{\mathrm{X}})\right]-\mathrm{d}_{3}\right\}+\left[\left(\mathrm{sqp}_{1}{ }^{2} \overline{\mathrm{X}}^{2}\right) /\left((\mathrm{s}+\overline{\mathrm{X}})^{3}\right)\right]>0
\end{gathered}
$$

Proof: Consider the Variation matrix (17) at disease-free equilibrium point $\bar{E}(\bar{X}, 0, \bar{Y}, 0,0)$ such that $V(\bar{E})=$ 


$$
\left(\begin{array}{ccccc}
\mathrm{r}-[(2 \mathrm{r} \overline{\mathrm{X}}) / \mathrm{k}]-\left[\left(\mathrm{sp}_{1} \overline{\mathrm{Y}}\right) /\left((\mathrm{s}+\overline{\mathrm{X}})^{2}\right)\right] & -[(\mathrm{r} \overline{\mathrm{X}}) / \mathrm{k}]-\beta \overline{\mathrm{X}} & -\left[\left(\mathrm{p}_{1} \overline{\mathrm{X}}\right) /(\mathrm{s}+\overline{\mathrm{X}})\right] & -\left[\left(\mathrm{p}_{3} \overline{\mathrm{X}}\right) /(\mathrm{s}+\overline{\mathrm{X}})\right] & \mathrm{r}_{1} \\
0 & \beta \overline{\mathrm{X}}-\mathrm{t}_{1}-\mathrm{d}_{2}-\frac{\mathrm{sp}_{2} \overline{\mathrm{Y}}}{\mathrm{s}^{2}} & 0 & 0 & 0 \\
{\left[\left(\mathrm{sp}_{1} \overline{\mathrm{Y}}\right) /\left((\mathrm{s}+\overline{\mathrm{X}})^{2}\right)\right]} & {\left[\left(\mathrm{sqp}_{2} \overline{\mathrm{Y}}\right) /\left((\mathrm{s}+\overline{\mathrm{X}})^{2}\right)\right]} & {\left[\left(\mathrm{qp}_{1} \overline{\mathrm{X}}\right) /(\mathrm{s}+\overline{\mathrm{X}})\right]-\mathrm{d}_{3}} & -\alpha \overline{\mathrm{Y}}-\mathrm{d}_{3} & \mathrm{r}_{2} \\
0 & 0 & 0 & {\left[\left(\mathrm{qp}_{3} \overline{\mathrm{X}}\right) /(\mathrm{s}+\overline{\mathrm{X}})\right]-\alpha \overline{\mathrm{Y}}-\mathrm{t}_{2}-\mathrm{d}_{4}} & 0 \\
0 & \mathrm{t}_{1} & 0 & \mathrm{t}_{2} & -\mathrm{d}_{1}-\mathrm{r}_{1}-\mathrm{r}_{2}
\end{array}\right)
$$

Then it is possible to find the determinant of the variation matrix as $\operatorname{Det}\left(V(\bar{E})-\lambda I_{5}\right)$

$$
\begin{aligned}
& \begin{array}{|ccccc}
\mathrm{r}-[(2 \mathrm{r} \overline{\mathrm{X}}) / \mathrm{k}]-\left[\left(\mathrm{sp}_{1} \overline{\mathrm{Y}}\right) /\left((\mathrm{s}+\overline{\mathrm{X}})^{2}\right)\right]-\lambda & -[(\mathrm{r} \overline{\mathrm{X}}) / \mathrm{k}]-\beta \overline{\mathrm{X}} & -\left[\left(\mathrm{p}_{1} \overline{\mathrm{X}}\right) /(\mathrm{s}+\overline{\mathrm{X}})\right] & -\left[\left(\mathrm{p}_{3} \overline{\mathrm{X}}\right) /(\mathrm{s}+\overline{\mathrm{X}})\right] & \mathrm{r}_{1} \\
0 & \beta \overline{\mathrm{X}}-\mathrm{t}_{1}-\mathrm{d}_{2}-\frac{\mathrm{sp}_{\mathrm{Y}} \mathrm{Y}}{\mathrm{s}^{2}}-\lambda & 0 & 0 & 0 \\
{\left[\left(\mathrm{sp}_{1} \overline{\mathrm{Y}}\right) /\left((\mathrm{s}+\overline{\mathrm{X}})^{2}\right)\right]} & {\left[\left(\mathrm{sqp}_{2} \overline{\mathrm{Y}}\right) /\left((\mathrm{s}+\overline{\mathrm{X}})^{2}\right)\right]} & {\left[\left(\mathrm{qp}_{1} \overline{\mathrm{X}}\right) /(\mathrm{s}+\overline{\mathrm{X}})\right]-\mathrm{d}_{3}-\lambda} & -\alpha \overline{\mathrm{Y}}-\mathrm{d}_{3} & \mathrm{r}_{2} \\
0 & 0 & 0 & {\left[\left(\mathrm{qp}_{3} \overline{\mathrm{X}}\right) /(\mathrm{s}+\overline{\mathrm{X}})\right]-\alpha \overline{\mathrm{Y}}-\mathrm{t}_{2}-\mathrm{d}_{4}-\lambda} & 0 \\
0 & \mathrm{t}_{1} & 0 & \mathrm{t}_{2} & -\mathrm{d}_{1}-\mathrm{r}_{1}-\mathrm{r}_{2}-\lambda
\end{array} \\
& \left(\beta \overline{\mathrm{X}}-\mathrm{t}_{1}-\mathrm{d}_{2}-\left[\left(\mathrm{sp}_{2} \overline{\mathrm{Y}}\right) /\left(\mathrm{s}^{2}\right)\right]-\lambda\right) *\left(\left[\left(\mathrm{qp}_{3} \overline{\mathrm{X}}\right) /(\mathrm{s}+\overline{\mathrm{X}})\right]-\alpha \overline{\mathrm{Y}}-\mathrm{t}_{2}-\mathrm{d}_{4}-\lambda\right) *\left(-\mathrm{d}_{1}-\mathrm{r}_{1}-\mathrm{r}_{2}-\lambda\right) * \\
& \left\{\left(\mathrm{r}-[(2 \mathrm{r} \overline{\mathrm{X}}) / \mathrm{k}]-\left[\left(\mathrm{sp}_{1} \overline{\mathrm{Y}}\right) /\left((\mathrm{s}+\overline{\mathrm{X}})^{2}\right)\right]-\lambda\right) *\left(\left[\left(\mathrm{qp}_{1} \overline{\mathrm{X}}\right) /(\mathrm{s}+\overline{\mathrm{X}})\right]-\mathrm{d}_{3}-\lambda\right)+\left[\left(\mathrm{sqp}_{1}{ }^{2} \overline{\mathrm{X}}^{2}\right) /\left((\mathrm{s}+\overline{\mathrm{X}})^{3}\right)\right]\right\}=0
\end{aligned}
$$

Eigen values are:

$$
\lambda_{1}=\beta \overline{\mathrm{X}}-\mathrm{t}_{1}-\mathrm{d}_{2}-\left[\left(\mathrm{sp}_{2} \overline{\mathrm{Y}}\right) /\left(\mathrm{s}^{2}\right)\right], \lambda_{2}=\left[\left(\mathrm{qp}_{3} \overline{\mathrm{X}}\right) /(\mathrm{s}+\overline{\mathrm{X}})\right]-\alpha \overline{\mathrm{Y}}-\mathrm{t}_{2}-\mathrm{d}_{4}, \lambda_{3}=-\mathrm{d}_{1}-\mathrm{r}_{1}-\mathrm{r}_{2}
$$

and the remaining eigen values can be obtained from the roots of quadratic equation:

$$
(\underbrace{\mathrm{r}-[(2 \mathrm{r} \overline{\mathrm{X}}) / \mathrm{k}]-\left[\left(\mathrm{sp}_{1} \overline{\mathrm{Y}}\right) /\left((\mathrm{s}+\overline{\mathrm{X}})^{2}\right)\right]}_{a}-\lambda)(\underbrace{\left[\left(\mathrm{qp}_{1} \overline{\mathrm{X}}\right) /(\mathrm{s}+\overline{\mathrm{X}})\right]-\mathrm{d}_{3}}_{b}-\lambda)+\underbrace{\left[\left(\mathrm{sq} \mathrm{p}_{1}{ }^{2} \overline{\mathrm{X}}^{2}\right) /\left((\mathrm{s}+\overline{\mathrm{X}})^{3}\right)\right]}_{c}=0
$$

It is known that a quadratic equation $(a-\lambda)(b-\lambda)+c=0$ is locally asymptotically stable iff $a+b>0 \& a b+c>$ 0, Using such Routh Hurwitz criterion, the disease free equilibrium point $\bar{E}(\overline{\mathrm{X}}, 0, \overline{\mathrm{Y}}, 0,0)$ is locallyasymptotically stable if $\{\mathrm{r}-$ $\left.[(2 \mathrm{r} \overline{\mathrm{X}}) / \mathrm{k}]-\left[\left(\mathrm{sp}_{1} \overline{\mathrm{Y}}\right) /\left((\mathrm{s}+\overline{\mathrm{X}})^{2}\right)\right]\right\}+\left\{\left[\left(\mathrm{qp}_{1} \overline{\mathrm{X}}\right) /(\mathrm{s}+\overline{\mathrm{X}})\right]-\mathrm{d}_{3}\right\}>0$,

$$
\&\left\{\mathrm{r}-[(2 \mathrm{r} \overline{\mathrm{X}}) / \mathrm{k}]-\left[\left(\mathrm{sp}_{1} \overline{\mathrm{Y}}\right) /\left((\mathrm{s}+\overline{\mathrm{X}})^{2}\right)\right]\right\} *\left\{\left[\left(\mathrm{qp}_{1} \overline{\mathrm{X}}\right) /(\mathrm{s}+\overline{\mathrm{X}})\right]-\mathrm{d}_{3}\right\}+\left(\mathrm{sq} \mathrm{p}_{1}{ }^{2} \overline{\mathrm{X}}^{2}\right) /\left((\mathrm{s}+\overline{\mathrm{X}})^{3}\right)>0
$$

$\lambda_{1}=\beta \overline{\mathrm{X}}-\mathrm{t}_{1}-\mathrm{d}_{2}-\left[\left(\mathrm{sp}_{2} \overline{\mathrm{Y}}\right) /\left(\mathrm{s}^{2}\right)\right] \leq 0, \& \lambda_{2}=\left[\left(\mathrm{qp}_{3} \overline{\mathrm{X}}\right) /(\mathrm{s}+\overline{\mathrm{X}})\right]-\alpha \bar{Y}-\mathrm{t}_{2}-\mathrm{d}_{4} \leq 0$ where, $\bar{X}=\left\{\left(\mathrm{d}_{3} \mathrm{~s}\right) /\left(\mathrm{qp}_{1}-\mathrm{d}_{3}\right)\right\} \& \bar{Y}=$ $\left[\left(\mathrm{rsq}\left(\mathrm{kqp}_{1}-\mathrm{kd}_{3}-\mathrm{d}_{3} \mathrm{~s}\right)\right) /\left(\left(\mathrm{qp}_{1}-\mathrm{d}_{3}\right)^{2} \mathrm{k}\right)\right]$, Otherwise the disease free equilibrium point is asymptotically unstable. Now let see again, the Global stability analysis of model(2) around the endemic equilibrium point or positive equilibrium point $E^{*}\left(X^{*}, W^{*}, Y^{*}, Z^{*}, H^{*}\right)$ which showsco-existence. For that let us state following theorem and prove by taking appropriate Liapunove function L.

Theorem 4.10[Global stability] Endemic Equilibrium point $E^{*}\left(X^{*}, W^{*}, Y^{*}, Z^{*}, H^{*}\right)$ exists and globally asymptotically stable.

Proof: Define appropriate Liapunove function

$L(X, W, \quad Y, \quad Z, \quad H)=\left\{\left[\left(X-X^{*}\right)^{2}\right] / 2\right\}+\left\{\left[\alpha_{1}\left(W-W^{*}\right)^{2}\right] /[2]\right\}+\left\{\left[\alpha_{2}\left(Y-Y^{*}\right)^{2}\right] /[2]\right\}+\left\{\left[\alpha_{3}\left(Z-Z^{*}\right)^{2}\right] /[2]\right\}+$ $\left\{\left[\left(H-H^{*}\right)^{2}\right] /[2]\right\}$. where $\alpha_{1}, \alpha_{2}, \alpha_{3}, \alpha_{4}>0$ are chosen properly such that, $d L / d t=0 \forall\left(X^{*}, W^{*}, Y^{*}, Z^{*}, H^{*}\right) \in \mathbb{R}_{+}^{5}$ and $d L / d t \leq 0 \forall(X, W, Y, Z, H) \in \mathbb{R}_{+}^{5}$. This implies $E^{*}$ of the system is Liapunove stable and $d L / d t<0, \forall(X, W, Y, Z, H) \in \mathbb{R}_{+}^{5}$ near $E^{*}$. This implies $E^{*}$ is globally asymptotically stable point. Now differentiate the liapunove function $\mathrm{L}$ with respect oftimet as:

$$
d L / d t=\left(X-X^{*}\right)[d X / d t]+\alpha_{1}\left(W-W^{*}\right)[d W / d t]+\alpha_{2}\left(Y-Y^{*}\right)[d Y / d t]+\alpha_{3}\left(Z-Z^{*}\right)[d Z / d t]+\alpha_{4}\left(H-H^{*}\right)[d H / d t]
$$

Now substitute the model (6)-(10) into (18), we have the following equation

$$
\begin{aligned}
& \mathrm{dL} / \mathrm{dt}=\left(X-X^{*}\right)\left[\mathrm{rX}(1-[\mathrm{X}+\mathrm{W}] / \mathrm{k})+\mathrm{r}_{1} \mathrm{H}-\beta \mathrm{XW}-\left[\mathrm{p}_{1} \mathrm{XY}\right] /[\mathrm{s}+\mathrm{X}]-\left[\mathrm{p}_{3} \mathrm{XZ}\right] /[\mathrm{s}+\mathrm{X}]\right] \\
& +\alpha_{1}\left(W-W^{*}\right)\left[\beta \mathrm{XW}-\mathrm{t}_{1} \mathrm{~W}-\mathrm{d}_{2} \mathrm{~W}-\left[\mathrm{p}_{2} \mathrm{WY}\right] /[\mathrm{s}+\mathrm{W}]-\left[\mathrm{p}_{4} \mathrm{WZ}\right] /[\mathrm{s}+\mathrm{W}]\right] \\
& +\alpha_{2}\left(Y-Y^{*}\right)\left[\left[\mathrm{qp}_{1} \mathrm{XY}\right] /[\mathrm{s}+\mathrm{X}]+\left[\mathrm{qp}_{2} \mathrm{WY}\right] /[\mathrm{s}+\mathrm{W}]+\mathrm{r}_{2} \mathrm{H}-\alpha \mathrm{YZ}-\mathrm{d}_{3} \mathrm{Y}\right] \\
& +\alpha_{3}\left(Z-Z^{*}\right)\left[\left[\mathrm{qp}_{3} \mathrm{XZ}\right] /[\mathrm{s}+\mathrm{X}]+\left[\mathrm{qp}_{4} \mathrm{WZ}\right] /[\mathrm{s}+\mathrm{W}]+\alpha \mathrm{YZ}-\mathrm{t}_{2} \mathrm{Z}-\mathrm{d}_{4} \mathrm{Z}\right] \\
& +\alpha_{4}\left(H-H^{*}\right)\left[\mathrm{t}_{1} \mathrm{~W}+\mathrm{t}_{2} \mathrm{Z}-d_{1} \mathrm{H}-\mathrm{r}_{1} \mathrm{H}-\mathrm{r}_{2} \mathrm{H}\right]
\end{aligned}
$$

Take out $X, W, Y, Z, H$ from each bracket and write a change as follows $\mathrm{dL} / \mathrm{dt}=\left(X-X^{*}\right)\left(X-X^{*}\right)\left[\mathrm{r}(1-[(\mathrm{X}+\mathrm{W}) / \mathrm{k}])+\left[\left(\mathrm{r}_{1} \mathrm{H}\right) / \mathrm{X}\right]-\beta \mathrm{W}-\left[\left(\mathrm{p}_{1} \mathrm{Y}\right) /(\mathrm{s}+\mathrm{X})\right]-\left[\left(\mathrm{p}_{3} \mathrm{Z}\right) /(\mathrm{s}+\mathrm{X})\right]\right]+$ $\alpha_{1}\left(W-W^{*}\right)\left(W-W^{*}\right)\left[\beta \mathrm{X}-\mathrm{t}_{1}-\mathrm{d}_{2}-\left[\left(\mathrm{p}_{2} \mathrm{Y}\right) /(\mathrm{s}+\mathrm{W})\right]-\left[\left(\mathrm{p}_{4} \mathrm{Z}\right) /(\mathrm{s}+\mathrm{W})\right]\right]+\alpha_{2}\left(Y-Y^{*}\right)\left(Y-Y^{*}\right)\left[\left[\left(\mathrm{qp}_{1} \mathrm{X}\right) /(\mathrm{s}+\mathrm{X})\right]+\right.$ 
$\left.\left[\left(\mathrm{qp}_{2} \mathrm{~W}\right) /(\mathrm{s}+\mathrm{W})\right]+\left[\left(\mathrm{r}_{2} \mathrm{H}\right) / \mathrm{Y}\right]-\alpha \mathrm{Z}-\mathrm{d}_{3}\right]+\alpha_{3}\left(Z-Z^{*}\right)\left(Z-Z^{*}\right)\left[\left[\left(\mathrm{qp}_{3} \mathrm{X}\right) /(\mathrm{s}+\mathrm{X})\right]+\left[\left(\mathrm{qp}_{4} \mathrm{~W}\right) /(\mathrm{s}+\mathrm{W})\right]+\alpha \mathrm{Y}-\mathrm{t}_{2}-\right.$ $\left.\mathrm{d}_{4}\right]+\alpha_{4}\left(H-H^{*}\right)\left(H-H^{*}\right)\left[\left[\left(\mathrm{t}_{1} \mathrm{~W}\right) / H\right]+\left[\left(\mathrm{t}_{2} \mathrm{Z}\right) / H\right]-\mathrm{d}_{1}-\mathrm{r}_{1}-\mathrm{r}_{2}\right]$, By rearranging, it is obtain that:

$\mathrm{dL} / \mathrm{dt}=-\left(X-X^{*}\right)^{2}\left[-\mathrm{r}(1-[(\mathrm{X}+\mathrm{W}) / \mathrm{k}])-\left[\left(\mathrm{r}_{1} \mathrm{H}\right) / \mathrm{X}\right]+\beta \mathrm{W}+\left[\left(\mathrm{p}_{1} \mathrm{Y}\right) /(\mathrm{s}+\mathrm{X})\right]+\left[\left(\mathrm{p}_{3} \mathrm{Z}\right) /(\mathrm{s}+\mathrm{X})\right]\right]-\alpha_{1}\left(I-I^{*}\right)^{2}\left[-\beta \mathrm{X}+\mathrm{t}_{1}+\mathrm{d}_{2}+\right.$ $\left.\left[\left(\mathrm{p}_{2} \mathrm{Y}\right) /(\mathrm{s}+\mathrm{W})\right]+\left[\left(\mathrm{p}_{4} \mathrm{Z}\right) /(\mathrm{s}+\mathrm{W})\right]\right]-\alpha_{2}\left(Y-Y^{*}\right)^{2}\left[-\left[\left(\mathrm{qp}_{2} \mathrm{~W}\right) /(\mathrm{s}+\mathrm{W})\right]-\left[\left(\mathrm{r}_{2} \mathrm{H}\right) / \mathrm{Y}\right]+\alpha \mathrm{Z}+\mathrm{d}_{3}\right]-$

$\alpha_{3}\left(Z-Z^{*}\right)^{2}\left[-\left[\left(\mathrm{qp}_{3} \mathrm{X}\right) /(\mathrm{s}+\mathrm{X})\right]-\left[\left(\mathrm{qp}_{4} \mathrm{~W}\right) /(\mathrm{s}+\mathrm{W})\right]-\alpha \mathrm{Y}+\mathrm{t}_{2}+\mathrm{d}_{4}\right]-\alpha_{4}\left(H-H^{*}\right)^{2}\left[-\left[\left(\mathrm{t}_{1} \mathrm{~W}\right) / H\right]-\left[\left(\mathrm{t}_{2} \mathrm{Z}\right) / H\right]+\mathrm{d}_{1}+\mathrm{r}_{1}+\mathrm{r}_{2}\right]$.Thus it is possible to $\operatorname{set} \alpha_{1}, \alpha_{2}, \alpha_{3}, \alpha_{4}$ such thatdL/dt $\leq 0$ and endemic equilibrium point $E^{*}$ is globally stable point.

\section{Basic Reproduction Number}

The basic reproduction number denoted by $\mathrm{R}_{0}$ and defined as the expected number of people getting secondary infection among the whole susceptible population. This number shows a potential for spread of disease within a given population. When $\mathrm{R}_{0}<1$ each infected individual produces on average less than one new infected individual so that the disease is expected to die out. On the other hand if $R_{0}>1$, then each individual produces more than one new infected individual so that the disease is expected to continue spreading in the population

Theorem 5.1 The basic reproduction number for infected prey at Disease free equilibrium point(DFEP)

$$
\begin{gathered}
\bar{E}(\bar{X}, 0, \bar{Y}, 0,0)=\left(\left[\mathrm{d}_{3} \mathrm{~s}\right] /\left[\mathrm{qp}_{1}-\mathrm{d}_{3}\right], \quad 0, \quad\left[\mathrm{rsq}\left(\mathrm{kqp}_{1}-\mathrm{kd}_{3}-\mathrm{d}_{3} \mathrm{~s}\right)\right] /\left[\left(\mathrm{qp}_{1}-\mathrm{d}_{3}\right)^{2} \mathrm{k}\right], \quad 0, \quad 0\right) \text { is given by } \\
R_{01}=\left[\left(\mathrm{qp}_{1}-\mathrm{d}_{3}\right)^{2} \mathrm{k} \mathrm{d}_{3} \mathrm{~s}^{2}\right] /\left[\left(\mathrm{qp}_{1}-\mathrm{d}_{3}\right)\left\{\left(\mathrm{qp}_{1}-\mathrm{d}_{3}\right)^{2} \mathrm{ks}\left(\mathrm{t}_{1}+\mathrm{d}_{2}\right)+\mathrm{rsqp}_{2}\left(\mathrm{kqp}_{1}-\mathrm{kd}_{3}-\mathrm{d}_{3} \mathrm{~s}\right)\right\}\right]
\end{gathered}
$$

Proof: Consider infected prey equation in (7):

$\mathrm{dW} / \mathrm{dt}=\beta \mathrm{XW}-\mathrm{t}_{1} \mathrm{~W}-\mathrm{d}_{2} \mathrm{~W}-\left[\mathrm{p}_{2} \mathrm{WY}\right] /[\mathrm{s}+\mathrm{W}]-\left[\mathrm{p}_{4} \mathrm{WZ}\right] /[\mathrm{s}+\mathrm{W}]=\left(\beta \mathrm{X}-\left[\mathrm{t}_{1}+\mathrm{d}_{2}+\left[\mathrm{p}_{2} \mathrm{Y}\right] /[\mathrm{s}+\mathrm{W}]+\left[\mathrm{p}_{4} \mathrm{Z}\right] /[\mathrm{s}+\mathrm{W}]\right]\right) \mathrm{W}$. Now let us define functions $\mathrm{F}$ and $\mathrm{V}, F=\beta \mathrm{X}, V=\mathrm{t}_{1}+\mathrm{d}_{2}+\left[\mathrm{p}_{2} \mathrm{Y}\right] /[\mathrm{s}+\mathrm{W}]+\left[\mathrm{p}_{4} \mathrm{Z}\right] /[\mathrm{s}+\mathrm{W}]$,Evaluate $\mathrm{F}$ and $\mathrm{V}$ at DFEP

$$
\begin{aligned}
& \bar{E}(\overline{\mathrm{X}}, 0, \overline{\mathrm{Y}}, 0,0)=\left(\left[\mathrm{d}_{3} \mathrm{~s}\right] /\left[\mathrm{qp}_{1}-\mathrm{d}_{3}\right], \quad 0, \quad\left[\mathrm{rsq}\left(\mathrm{kqp}_{1}-\mathrm{kd}_{3}-\mathrm{d}_{3} \mathrm{~s}\right)\right] /\left[\left(\mathrm{qp}_{1}-\mathrm{d}_{3}\right)^{2} \mathrm{k}\right], \quad 0,0\right) \\
& F(\bar{E})=\beta \overline{\mathrm{X}}=\left[\beta \mathrm{d}_{3} \mathrm{~s}\right] /\left[\mathrm{qp}_{1}-\mathrm{d}_{3}\right], V(\bar{E})=\mathrm{t}_{1}+\mathrm{d}_{2}+\left[\left(\mathrm{p}_{2} \overline{\mathrm{Y}}\right) / \mathrm{s}\right]=\mathrm{t}_{1}+\mathrm{d}_{2}+\left[\left(\mathrm{rsqp}_{2}\left(\mathrm{kqp}_{1}-\mathrm{kd}_{3}-\mathrm{d}_{3} \mathrm{~s}\right)\right) /\left(\left(\mathrm{qp}_{1}-\mathrm{d}_{3}\right)^{2} \mathrm{ks}\right)\right]
\end{aligned}
$$

which more simplified to $V(\bar{E})=\left\{\left[\left(\mathrm{qp}_{1}-\mathrm{d}_{3}\right)^{2}\left(\mathrm{t}_{1}+\mathrm{d}_{2}\right) \mathrm{ks}+\mathrm{rsqp}_{2}\left(\mathrm{kqp}_{1}-\mathrm{kd}_{3}-\mathrm{d}_{3} \mathrm{~s}\right)\right] /\left[\left(\mathrm{qp}_{1}-\mathrm{d}_{3}\right)^{2} \mathrm{ks}\right]\right\}$

Then the basic reproduction number of infected prey is

$$
\begin{gathered}
R_{01}=F V^{-1}=\left\{\left[\beta \mathrm{d}_{3} \mathrm{~s}\right] /\left[\mathrm{qp}_{1}-\mathrm{d}_{3}\right]\right\} *\left\{\left[\left(\mathrm{qp}_{1}-\mathrm{d}_{3}\right)^{2} \mathrm{ks}\right] /\left[\left(\mathrm{qp}_{1}-\mathrm{d}_{3}\right)^{2}\left(\mathrm{t}_{1}+\mathrm{d}_{2}\right) \mathrm{ks}+\mathrm{rsqp}_{2}\left(\mathrm{kqp}_{1}-\mathrm{kd}_{3}-\mathrm{d}_{3} \mathrm{~s}\right)\right]\right\} \\
R_{01}=\left\{\left[\left(\mathrm{qp}_{1}-\mathrm{d}_{3}\right)^{2} \mathrm{k} \beta \mathrm{d}_{3} \mathrm{~s}^{2}\right] /\left[\left(\mathrm{qp}_{1}-\mathrm{d}_{3}\right)\left\{\left(\mathrm{qp}_{1}-\mathrm{d}_{3}\right)^{2}\left(\mathrm{t}_{1}+\mathrm{d}_{2}\right) \mathrm{ks}+\mathrm{rsqp}_{2}\left(\mathrm{kqp}_{1}-\mathrm{kd}_{3}-\mathrm{d}_{3} \mathrm{~s}\right)\right\}\right]\right\}
\end{gathered}
$$

Theorem 5.2. The basic reproduction number for infected predators at Disease-free equilibrium point (DFEP)

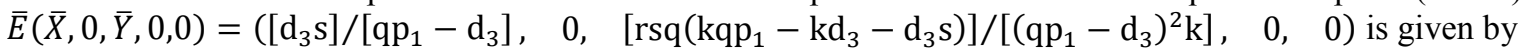

$$
R_{02}=\left\{\left[\left(\mathrm{qp}_{1}-\mathrm{d}_{3}\right)\left(\mathrm{qp}_{3} \mathrm{~d}_{3}\right) \mathrm{k}+\alpha \mathrm{rsq}\left(\mathrm{kqp}_{1}-\mathrm{kd}_{3}-\mathrm{d}_{3} \mathrm{~s}\right)\right] /\left[\left(\mathrm{qp}_{1}-\mathrm{d}_{3}\right)^{2}\left(\mathrm{t}_{2}+\mathrm{d}_{4}\right) \mathrm{k}\right]\right\}
$$

Proof: Consider the infected predator model equation in (9):

$\frac{\mathrm{dZ}}{\mathrm{dt}}=\left[\mathrm{qp}_{3} \mathrm{XZ}\right] /[\mathrm{s}+\mathrm{X}]+\left[\mathrm{qp}_{4} \mathrm{WZ}\right] /[\mathrm{s}+\mathrm{W}]+\alpha \mathrm{YZ}-\mathrm{t}_{2} \mathrm{Z}-\mathrm{d}_{4} \mathrm{Z}=\left\{\left[\mathrm{qp}_{3} \mathrm{X}\right] /[\mathrm{s}+\mathrm{X}]+\left[\mathrm{qp}_{4} \mathrm{~W}\right] /[\mathrm{s}+\mathrm{W}]+\alpha \mathrm{Y}-\right.$

$\left.\left(t_{2}+d_{4}\right)\right\} Z$. Now let us define functions $F \& V$ as follows:

$F=\left[\mathrm{qp}_{3} \mathrm{X}\right] /[\mathrm{s}+\mathrm{X}]+\left[\mathrm{qp}_{4} \mathrm{~W}\right] /[\mathrm{s}+\mathrm{W}]+\alpha \mathrm{Y}$, and $\mathrm{V}=\mathrm{t}_{2}+\mathrm{d}_{4}$ Then Evaluate $F$ and $V$ at (DFEP) $\bar{E}(\bar{X}, 0, \bar{Y}, 0,0)=$

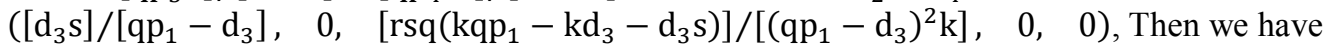

$$
\begin{aligned}
& F(\bar{E})=\left\{\left[\mathrm{qp}_{3} \mathrm{~d}_{3} \mathrm{~s}\right] /\left[\mathrm{s}\left(\mathrm{qp}_{1}-\mathrm{d}_{3}\right)\right]\right\}+\left\{\left[\alpha \mathrm{rsq}\left(\mathrm{kqp}_{1}-\mathrm{kd}_{3}-\mathrm{d}_{3} \mathrm{~s}\right)\right] /\left[\left(\mathrm{qp}_{1}-\mathrm{d}_{3}\right)^{2} \mathrm{k}\right]\right\} \\
= & {\left[\left(\mathrm{qp}_{1}-\mathrm{d}_{3}\right)\left(\mathrm{qp}_{3} \mathrm{~d}_{3}\right) \mathrm{k}+\alpha \mathrm{rsq}\left(\mathrm{kqp}_{1}-\mathrm{kd}_{3}-\mathrm{d}_{3} \mathrm{~s}\right)\right] /\left[\left(\mathrm{qp}_{1}-\mathrm{d}_{3}\right)^{2} \mathrm{k}\right], \text { and } \mathrm{V}(\bar{E})=\mathrm{t}_{2}+\mathrm{d}_{4} }
\end{aligned}
$$

Therefore the basic reproduction number of infected predator is $R_{02}=F V^{-1}$ and hence

$$
R_{02}=\left\{\left[\left(\mathrm{qp}_{1}-\mathrm{d}_{3}\right)\left(\mathrm{qp}_{3} \mathrm{~d}_{3}\right) \mathrm{k}+\alpha \mathrm{rsq}\left(\mathrm{kqp}_{1}-\mathrm{kd}_{3}-\mathrm{d}_{3} \mathrm{~s}\right)\right] /\left[\left(\mathrm{qp}_{1}-\mathrm{d}_{3}\right)^{2}\left(\mathrm{t}_{2}+\mathrm{d}_{4}\right) \mathrm{k}\right]\right\}
$$

\section{Simulation}

In this section, Simulation of model (6)-(10) is carried out using DEDiscover Version: 2.6.4. software. For Simulation, a set of meaningful values are assigned to model parameters and initial value for model variables are given in tables bellow. The model is arranged in such way for Simulation purposes.

$\mathrm{dX} / \mathrm{dt}=\mathrm{r} * \mathrm{X} *(1-(\mathrm{X}+\mathrm{W}) / \mathrm{k})+\mathrm{r} \_1 * \mathrm{H}-$ beta $^{*} \mathrm{X} * \mathrm{~W}-\mathrm{P} \_1 * \mathrm{X} * \mathrm{Y} /(\mathrm{s}+\mathrm{X})-\mathrm{P} \_3 * \mathrm{X} * \mathrm{Z} /(\mathrm{s}+\mathrm{X}) / /$ Susceptible prey

$\mathrm{dW} / \mathrm{dt}=$ beta $* \mathrm{X} * \mathrm{~W}-\mathrm{t} \_1 * \mathrm{~W}-\mathrm{d} \_2 * \mathrm{~W}-\mathrm{P} 22 * \mathrm{~W} * \mathrm{Y} /(\overline{\mathrm{s}}+\mathrm{W})-\mathrm{P} \_4 * \mathrm{~W} * \mathrm{Z} /(\mathrm{s}+\mathrm{W}) / /$ Infected prey

$\mathrm{dY} / \mathrm{dt}=\mathrm{q} * \mathrm{P} \_1 * \mathrm{X} * \mathrm{Y} /(\mathrm{s}+\mathrm{X})+\overline{\mathrm{q}} * \mathrm{P} \_2 * \overline{\mathrm{W}} * \mathrm{Y} /(\mathrm{s}+\mathrm{W})+\mathrm{r} \_2 * \overline{\mathrm{H}}-\mathrm{alpha} * \mathrm{Y} * \mathrm{Z}-\mathrm{d} \_3 * \mathrm{Y} / /$ Susceptible predator 
$\mathrm{dZ} / \mathrm{dt}=\mathrm{q} * \mathrm{P} \_3 * \mathrm{X} * \mathrm{Z} /(\mathrm{s}+\mathrm{X})+\mathrm{q} * \mathrm{P} \_4 * \mathrm{~W} * \mathrm{Z} /(\mathrm{s}+\mathrm{W})$-alpha*Y*Z-t_2*Z-d_4 $4 \mathrm{Z} / /$ Infected predator $\mathrm{dH} / \mathrm{dt}=\mathrm{t} \_1 * \mathrm{~W}+\mathrm{t} \_2 * \mathrm{Z}-\mathrm{d} \_1 * \mathrm{H}-\mathrm{r} \_1 * \mathrm{H}-\mathrm{r} \_2 * \mathrm{H} / /$ both infected populations under treatment

Table 4. Parameter value used for simulation.

\begin{tabular}{lll}
\hline Name & value & Description \\
\hline $\mathrm{r}$ & 22.4000 & growth rate of susceptible prey \\
$\mathrm{k}$ & $1.0000 \mathrm{E} 03$ & carrying capacity of susceptible prey \\
$\mathrm{r} \_1$ & 1.0000 & Recovery rate of \\
beta & 2.4000 & disease transmission rate in prey \\
$\mathrm{P} \_1$ & 1.0000 & predation coefficient of susceptible prey due to susceptible predator \\
$\mathrm{S}$ & 1.0000 & Half saturated rate \\
$\mathrm{P} \_3$ & 1.0000 & predation coefficient of susceptible prey due to infected predator \\
$\mathrm{t} \_1$ & 1.0000 & Treatment rate of infected prey \\
d_2 & 1.0000 & Death rate of infected prey \\
$\mathrm{P} \_2$ & 1.0000 & predation coefficient of infected prey due to predators \\
$\mathrm{P} \_4$ & 1.0000 & Predation rate of infected prey due to infected predator \\
$\mathrm{q}$ & 1.0000 & efficiency of predation \\
$\mathrm{r} \_2$ & 1.0000 & Recovery rate of susceptible predator \\
alpha & 2.6000 & Disease transmission rate in predator \\
d_3 & 1.0000 & Death rate of susceptible predator \\
t_2 & 1.0000 & Treatment rate of infected predator \\
d_4 & 1.0000 & Death rate of infected predator \\
d_1 & 1.0000 & Death rate of both infected and infected predator under treatment \\
\hline
\end{tabular}

Table 5. Initial Conditions used for model variables.

\begin{tabular}{lll}
\hline Name & value & Description \\
\hline $\mathrm{X}[\mathrm{t} 0]$ & $1.2000 \mathrm{E} 04$ & initial \# susceptible prey \\
$\mathrm{W}[\mathrm{t} 0]$ & 200.0000 & initial \# infected prey \\
$\mathrm{H}[\mathrm{t} 0]$ & 1.0000 & Initial \# under treated prey predator \\
$\mathrm{Y}[\mathrm{t} 0]$ & 160.0000 & initial \# susceptible predator \\
$\mathrm{Z}[\mathrm{t} 0]$ & 180.0000 & initial \# of infected predator \\
\hline
\end{tabular}

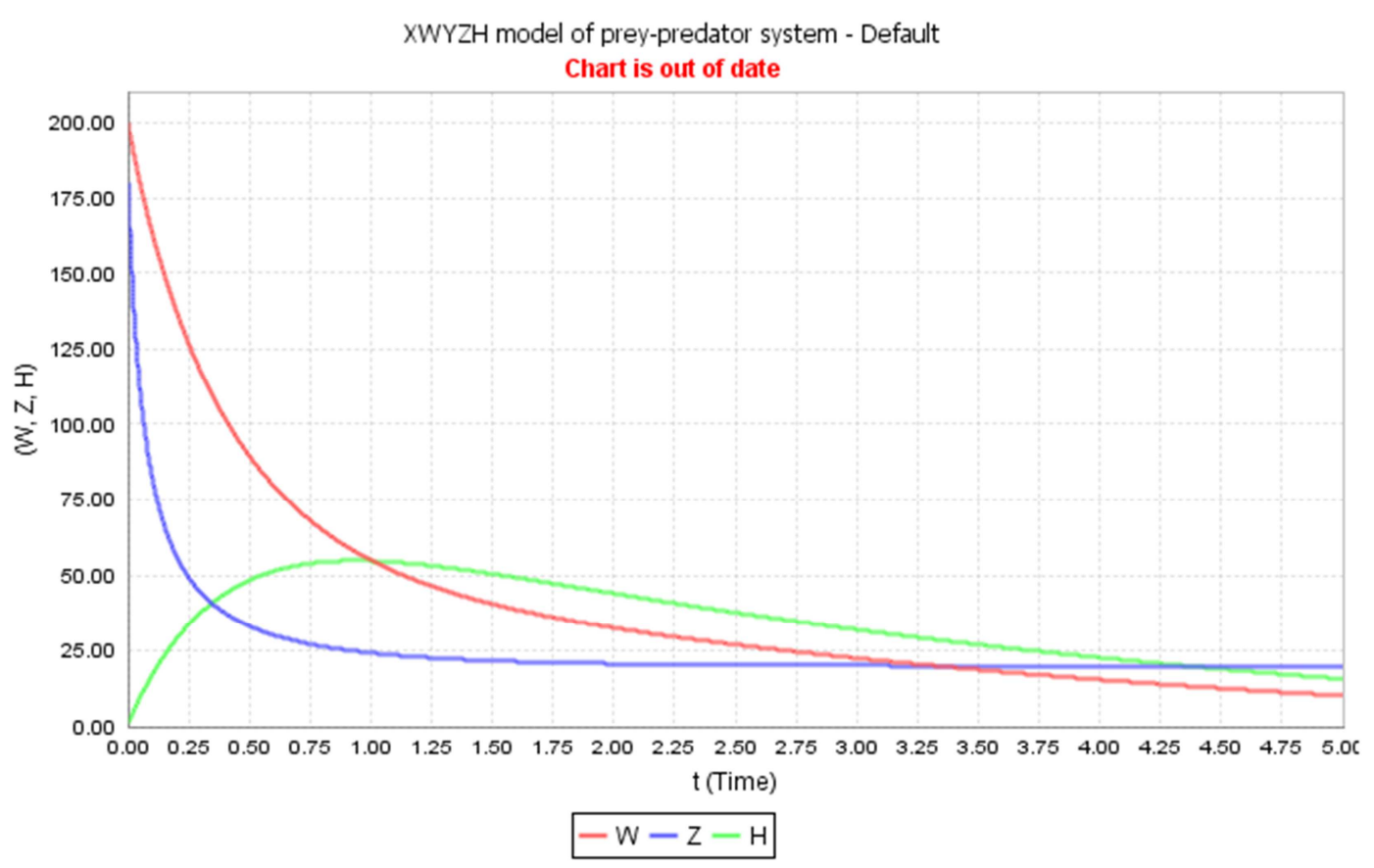

Figure 2. Infection prey-predator with treatment. 


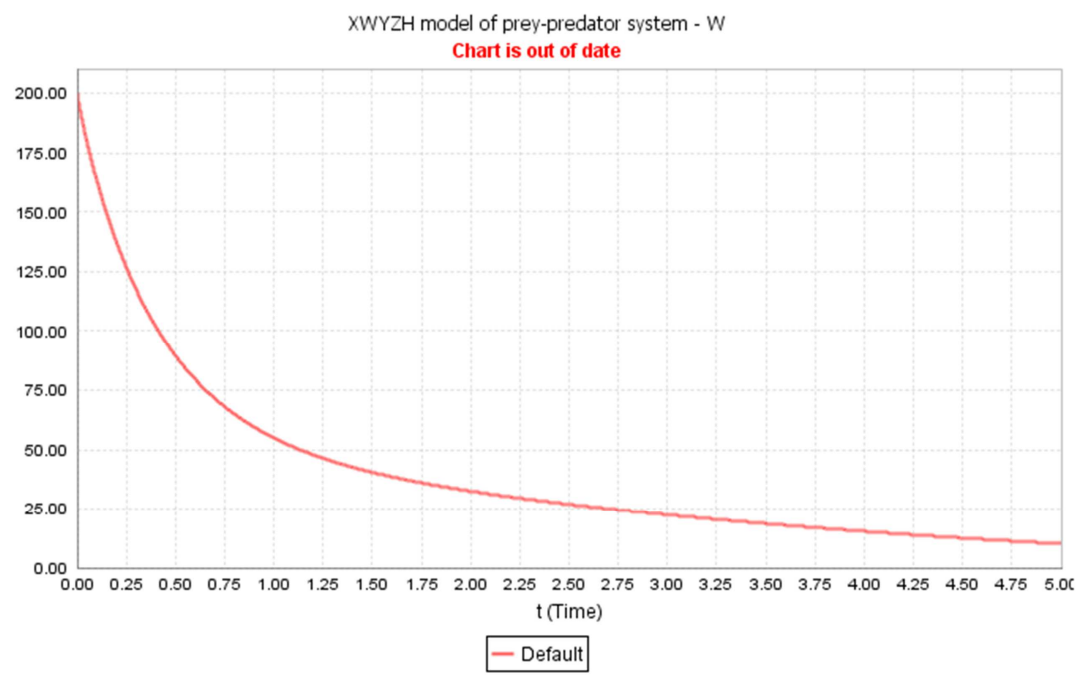

XWYZH model of prey-predator system - Z

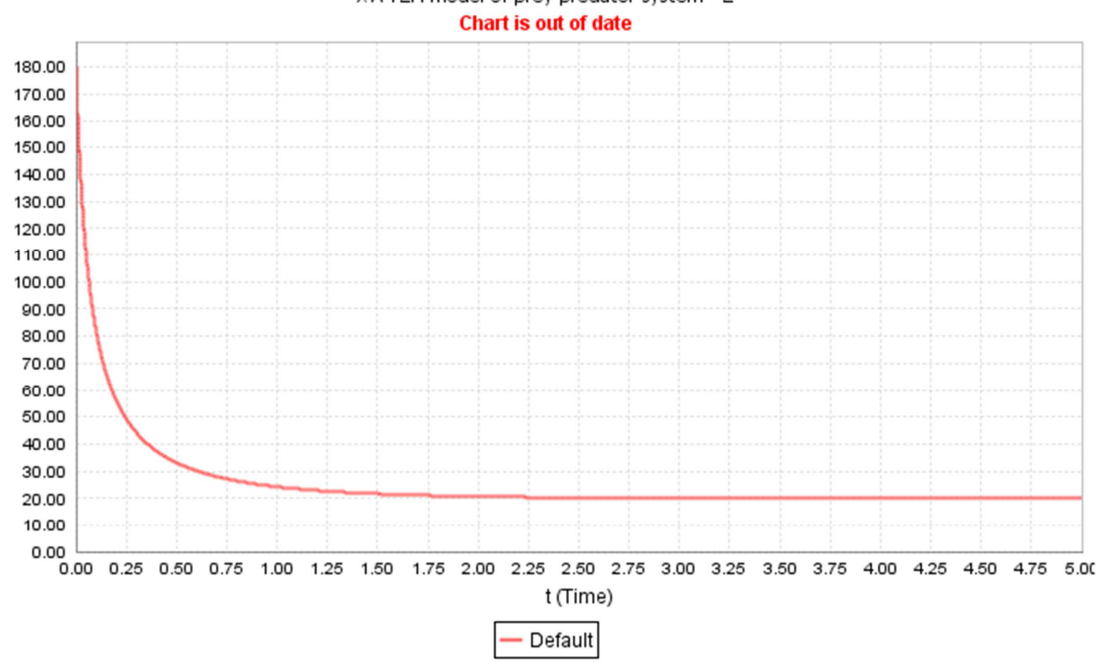

XWYZH model of prey-predator system - $\mathrm{H}$

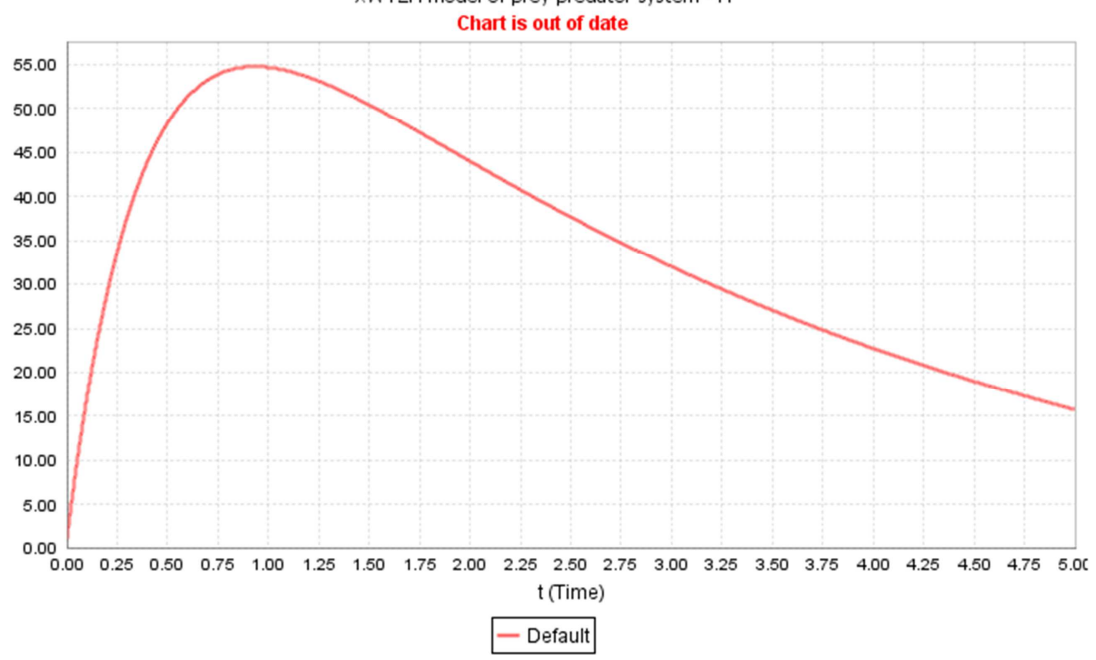

Figure 3. Individualplots for $W, Z$, and $H$.

From simulation Figure 2 and Figure 3, it can be conclude that treatment is helpful tool to minimize or eradicate infection. it is shown that as treatment rate increase on infected prey-predator, then infected prey-predator population decrease rapidly. This shows due to the fact that infected prey-predator population recovering and move to susceptible classes and that Contributes the susceptible prey-predator population to rise in number. 


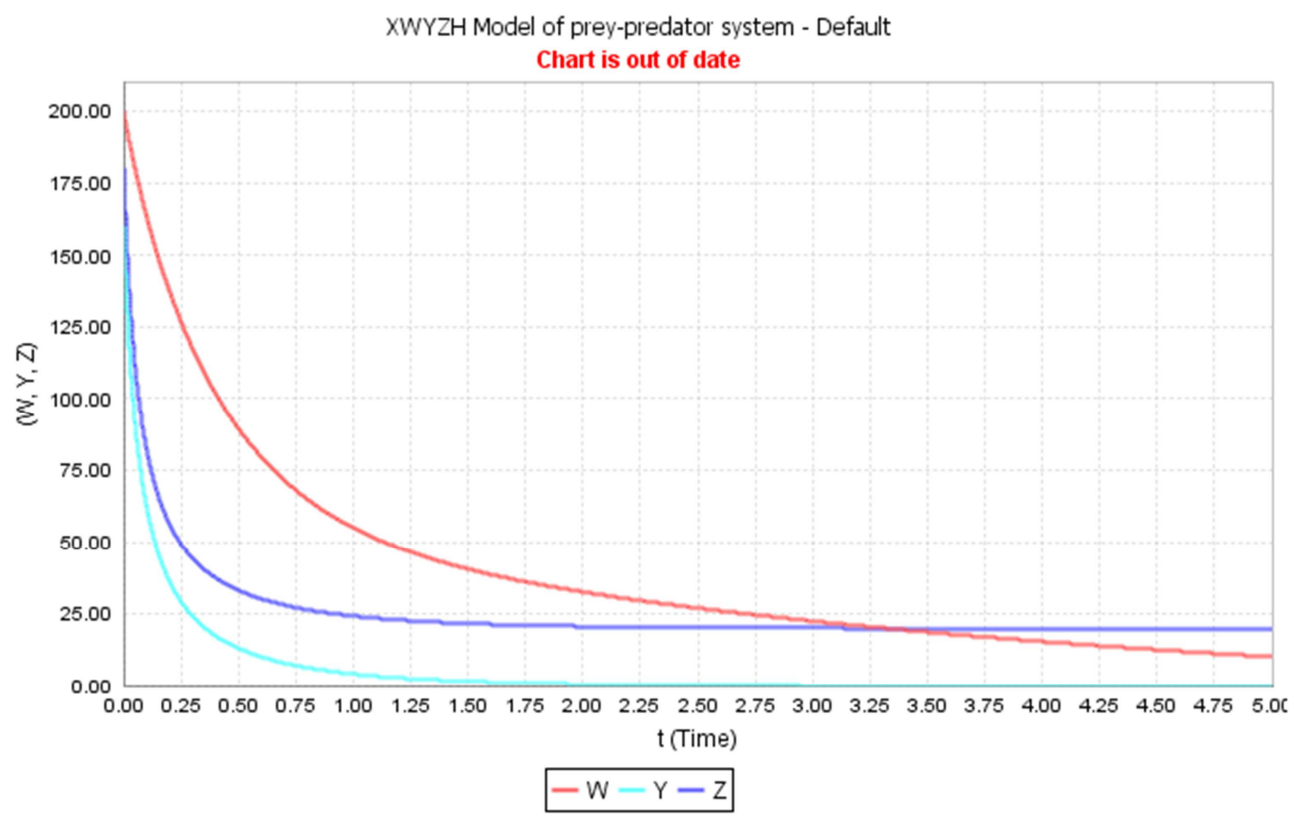

Figure 4. High infection and predation.

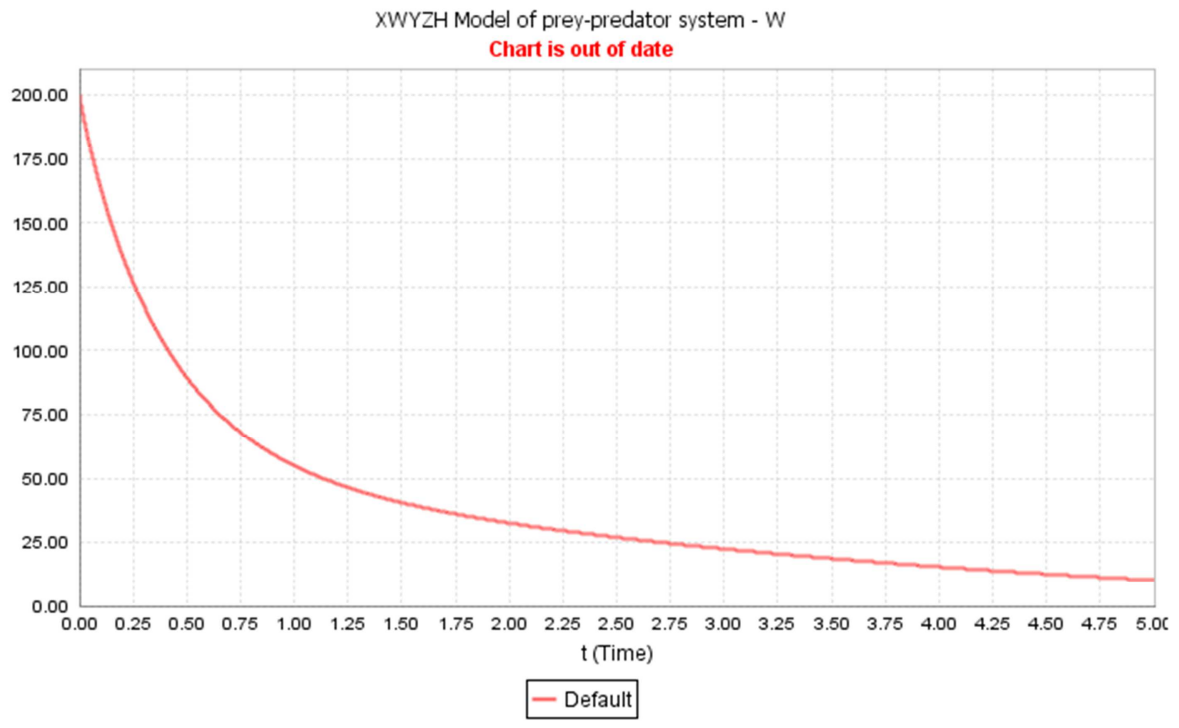

XWYZH Model of prey-predator system - Y

Chart is out of date

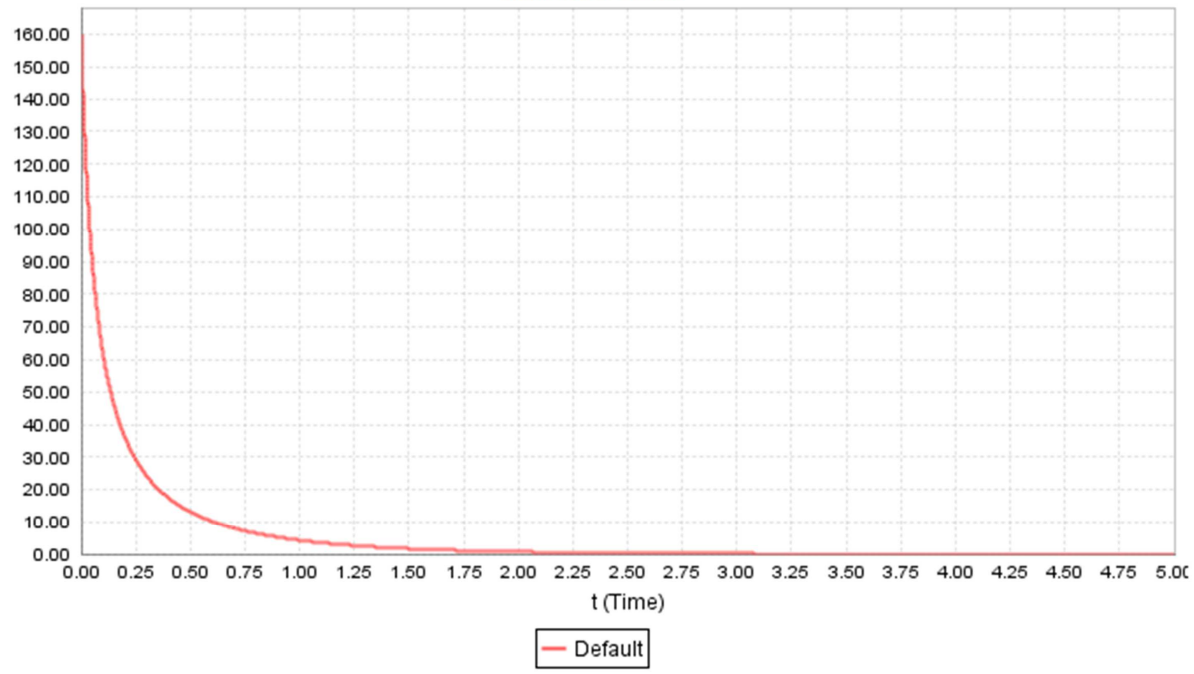




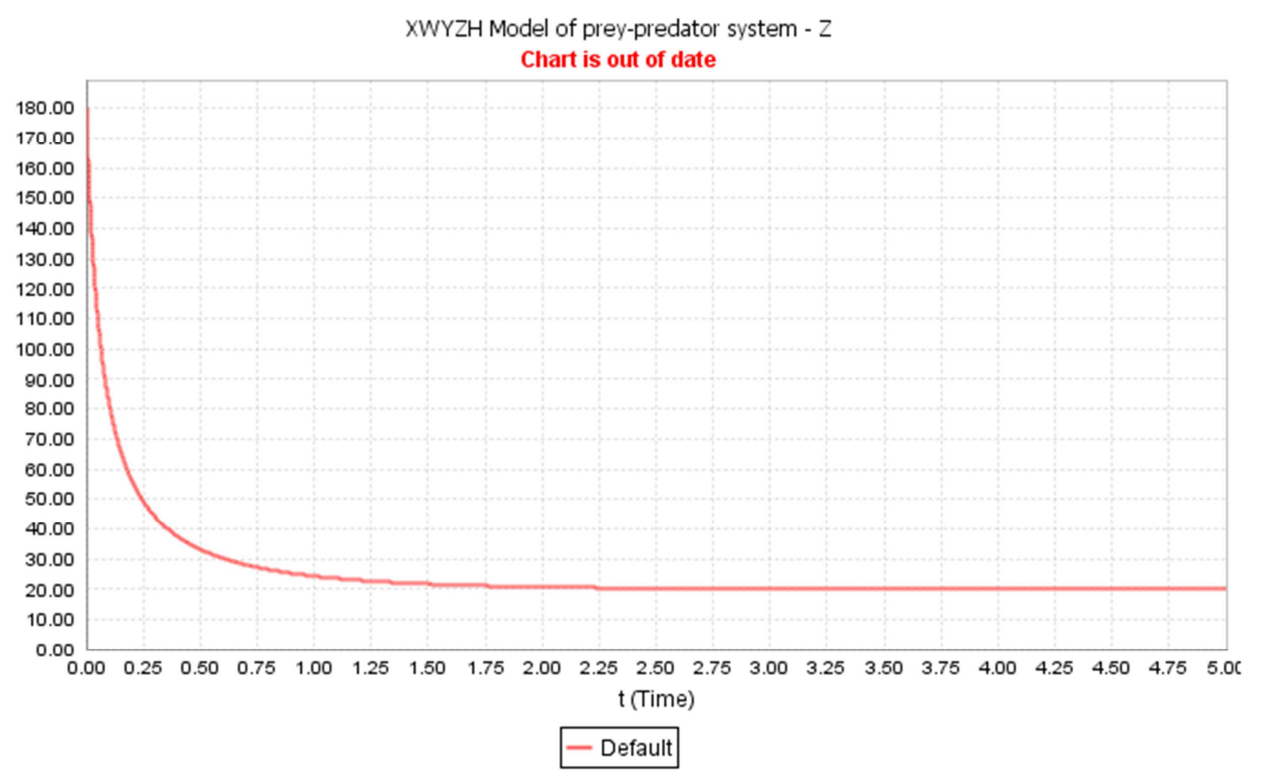

Figure 5. Individual plot of $W, Y$ and $Z$.

In sample Simulation figure 4 and figure 5 shows that high infection and predation results the whole prey-predator population decline to a certain level. Therefore it is better to implement treatment mechanisms to sustain stability of the prey-predator system.

\section{Conclusions and Recommendation}

In this paper, It can be concluded that the formulated model is Mathematically meaningful, valid, and biologically well posed by proving the boundedness, positivity and existence of the solutions of the model. Trivial, Axial, Disease-free and endemic Equilibrium points are investigated. Moreover, It is observed that in our model trivial equilibrium point is always locally asymptotically unstable. Axial equilibrium point is locally asymptotically stable if and only if the variables satisfy the following three conditions:(i) $\beta \mathrm{k}-$ $\left(\mathrm{t}_{1}+\mathrm{d}_{2}\right)<0$, (ii) $\mathrm{qp}_{1} \mathrm{k}-\mathrm{d}_{3}(\mathrm{~s}+\mathrm{k})<0$, \& (iii) $q \mathrm{p}_{3} \mathrm{k}-$ $\left(t_{2}+d_{4}\right)(s+k)<0$. Treatment is helpful tool to minimize or eradicate infection in prey-predator system. Therefore Providing treatment in infected prey-predator system creates opportunity to recover from illness and the prey-predator population can be saved and exist in stable situation. Thus, it is recommended to apply treatment on infected prey-predator to make the whole prey-predator population safe and abundant in nature. One can extend this paper by Assuming the predator grows logistically or by adding parameter like death rate on the prey or by including other variables like vaccination, immigration, migration on prey-predator system, and these things can be considered as limitation of this paper.

\section{References}

[1] Sachin Kumar and Harsha Kharbanda. Stability Analysis Of Prey-Predator Model With Infection, Migration and Vaccination In Prey, arXiv: 1709.10319vl [math.DS], 29 Sep 2017.
[2] Sudipa, Sinha, O.P. Misra, J. Dhar. Modeling a predator-prey system with infected prey in polluted environment., Elsevier, Applied Mathematics, April 2008, doi:10.1016/j,pm. 2009.10.003

[3] Rald Kamel Naji, Kawa Ahmed Hasan. The Dynamics of Prey-Predator Model With Disease In Prey, J. Math. Comput. Sci. 2 (2012), No. 4, 1052-1072, Available online at http://scik.org

[4] C. M. Silva (2017). Existence of periodic solutions for periodic eco-epidemic models with disease in the prey, J. Math. Anal. Appl. 453(1), 383-397.

[5] J. Chattopadhyay and O. Arino. A predator-prey model with disease in the prey, Nonlinear Analysis, 36 (1999), 747-766.

[6] A. F. Bezabih. Mathematical Eco-Epidemic Model on PreyPredator System. IOSR Journal of Mathematics (IOSR-JM), 16(1), (2020): pp. 22-34.

[7] A. F. Bezabih, G. K. Edessa, P. R. Koya. Mathematical EcoEpidemiological Model on Prey-Predator System. Mathematical Modeling and Applications. Vol. 5, No. 3, 2020, pp. 183-190. doi: 10.11648/j.mma.20200503.17

[8] A. F. Bezabih, G.K. Edessa, P. R. Koya. Mathematical Epidemiology Model Analysis on the Dynamics of COVID-19 Pandemic. American Journal of Applied Mathematics. Vol. 8, No. 5, 2020, pp. 247-256. doi: 10.11648/j.ajam.20200805.12.

[9] S. P. Bera, A. Maiti, G. Samanta. A Prey-predator Model with Infection in both prey and predator,Filomat 29:8 (2015),17531767.

[10] Asrul Sani, Edi Cahyono, Mukhsar, Gusti Arviana Rahman (2014). Dynamics of Disease Spread in a Predator-Prey System, Indonesia, Advanced Studies in Biology, Vol. 6, 2014, No. $4,169-179$.

[11] Alfred Hugo, Estomih S. Massawe, and Oluwole Daniel Makinde. An Eco-Epidemiological Mathematical Model with Treatment and Disease Infection in both Prey and Predator Population. Journal of Ecology and natural environment Vol. 4 (10), pp. 266-273, July 2012. 
[12] G. K. Edessa, B. Kumsa, P. R. Koya. Modeling and Simulation Study of the Population Dynamics of CommensalHost-Parasite System. American Journal of Applied Mathematics. Vol. 6, No. 3, 2018, pp. 97-108.

[13] S. Tolcha, B. Kumsa, P. R. Koya. Modeling and Simulation Study of Mutuality Interactions with Type II functional
Response and Harvesting. American Journal of Applied Mathematics. Vol. 6, No. 3, 2018, pp. 109-116.doi: 10.11648/j.ajam.20180603.

[14] M. Haque. A predator-prey model with disease in the predator species only, Nonlinear Anal., Real World Appl., 11(4) (2010), 2224-2236. 